\title{
Sakai-Sugimoto Brane System at High Density
}

\author{
D. Yamada \\ Racah Institute of Physics, The Hebrew University of Jerusalem, \\ Givat Ram, Jerusalem, 91904 Israel \\ daisuke@phys.huji.ac.il
}

\begin{abstract}
The D4-D8 brane system of Sakai-Sugimoto model at high quark density is studied in the weak coupling regime. We show that the color superconducting phase (for $N_{c} \approx 3$ ) or the chiral density wave (for $N_{c} \rightarrow \infty$ ) disappears at very large chemical potential, or equivalently at very large compactified dimension that the model possesses. We also comment on the prospects in the strong coupling regime along with the QCD phase diagram.
\end{abstract}

\section{Contents}

1 Introduction

2 Weak Coupling Field Theory Analysis 4 2.1 Qualitative Discussion . . . . . . . . . . . . . . . 4 2.1.1 BCS and DGR Instabilities . . . . . . . . . . . . . . 4

2.1.2 Sakai-Sugimoto Brane System at High Density . . . . . . . . . . . 6

2.2 Quantitative Discussion . . . . . . . . . . . . . . . . . 9

2.2.1 Renormalization Group Equations . . . . . . . . . . . . . . 9

2.2.2 Dyson-Schwinger Equations . . . . . . . . . . . . 15

3 Comments on the Strong Coupling Regime 21

A Some Formulas

B Solving Gap Equation 25 


\section{Introduction}

Decades have passed since quantum chromodynamics was accepted as the theory of strong interaction. While the successes of this theory are impressive, there still remain numerous unresolved problems. The most prominent of those problems is the confinement. At low energy, we observe hadrons instead of the QCD fundamental degrees of freedom and we still do not quite understand the mechanism of this phenomenon. The lack of the understanding is mainly due to the strongly coupled nature of the theory at low energy and to the fact that we do not have very good analytical control over the field theory in such coupling regime. The best hope, therefore, is that the numerical study could provide important insights into QCD at strong coupling.

Confinement is one of the phases of QCD and there are other phases in different regions of the QCD parameter space. One such example is the deconfined phase at high temperature. The numerical method, in fact, is proven to be powerful in the investigation of the confinement/deconfinement phase transition, and has provided the estimates of the transition temperature and the order of the phase transition. (See Reference [1] and the papers cited therein.) There is, however, a large region of the parameter space in which even the numerical study has not been very successful. Namely, the theory at finite quark (baryon) density. There is a problem in carrying out lattice simulations with the nonzero chemical potentials that are conjugate to the density and this is known as the sign problem (see e.g. Reference [2]). While the effort to overcome the sign problem vigorously continues, there have been some developments in analytic approaches. These include the analysis at asymptotically large chemical potential and also the use of NJL type models. The former takes the advantage that the QCD coupling is expected to be weak at very high density and perturbative computations from the QCD Lagrangian itself are possible. The latter models are pure fermionic and quartic couplings, which reproduce properties of QCD interactions in a certain degree, are introduced 1

What has been emerging from these analyses is the very rich phase structure of QCD in the parameter space of the temperature and the chemical potential. (See, for example, Reference [8] for the phase diagram.) In general, a cold and highly dense quark matter is expected to become a color superconductor [9]. As mentioned before, when the quark chemical potential $\mu$ is very large, the coupling $g(\mu)$ is small and the excitations near the Fermi surface of the quarks, particles and holes, are nearly free, and this naive ground state at high density is known as the Fermi liquid. However, the pairs of the particles at the antipodal points of the Fermi sphere are all degenerate and it costs no free energy to form such a pair. Then, if there is an attractive force between the particles (or holes), the pairing actually reduces the free energy of the system, leading to the instability of the naive ground state against the formation of the pair (Cooper pair). This is known as the Bardeen-Cooper-Schrieffer (BCS) instability [10] and in their original work for the electron gas in a solid, the attractive force was provided by the phonon exchange. For the case of high density QCD, there is an attractive force in a color channel and it leads to the similar BCS instability.

In particular, if we have three massless flavors in the theory, a very interesting form of

\footnotetext{
${ }^{1}$ A good modern review on perturbative high density QCD is Reference 1 . The NJL-model was introduced by Nambu and Jona-Lasinio [3, 4] as the model that exhibits the chiral symmetry breaking and light hadronic spectrum. A good review on the NJL model is Reference [5. The model is applied to QCD at finite density and reviewed in References [6, 17.
} 
the condensate has been found to form [11]2 This is the (scalar) diquark condensate of the form

$$
\left\langle q_{L i}^{a} q_{L j}^{b}\right\rangle=-\left\langle q_{R i}^{a} q_{R j}^{b}\right\rangle=\Delta_{C F L}\left(\delta_{i}^{a} \delta_{j}^{b}-\delta_{j}^{a} \delta_{i}^{b}\right),
$$

where the superscripts $a, b$ are the color indices, the subscripts $i, j$ are for the flavors, the subscripts $L, R$ indicates the chirality of the quarks and $\Delta_{C F L}$ is the size of the condensate (the gap). As one can see, the Kronecker deltas relate the flavor and color symmetries and those are not separately preserved by the condensate. The residual symmetry is the simultaneous flavor and (global) color rotations and for this reason, this phenomenon is called the color-flavor locking (CFL). The full symmetry breaking pattern of CFL is

$$
S U(3)_{\text {color }} \times S U(3)_{L} \times S U(3)_{R} \times U(1)_{V} \times U(1)_{E M} \rightarrow S U(3)_{\text {color }+L+R} \times \mathbb{Z}_{2} \times U(1)_{\tilde{Q}}
$$

where $S U(3)_{\text {color }+L+R}$ is the global diagonal subgroup of the original color and flavor symmetries, $\mathbb{Z}_{2}$ is the subgroup of $U(1)_{V}$ that changes the sign of all the quarks and $U(1)_{\tilde{Q}}$ is known as the "modified electromagnetism" whose gauge boson is a linear combination of the original photon and one of the gluons [11, 1. We observe that since $L$ - and $R$-flavor symmetries both lock to the color, the chiral symmetry is broken through the color factor. Even though the mechanism of the chiral symmetry breaking is very unusual, the corresponding chiral Lagrangian can be built [12]. This novel phase of QCD created a renewed and wide interest in the QCD phase structure and many generalizations and modifications have been explored. For example, when one considers finite quark masses, there are other possible forms of the condensate and those can be energetically favored for some regions of the $\mu$-T parameter space, resulting in the complicated structure of the phase diagram. The interested reader can pursuit the subject in the review papers cited above.

Rather than continuing to overview the QCD phase structure, we would now like to turn to the high density behavior in the 't Hooft limit with weak coupling. (This is not QCD, which has $N_{c}=3$, but has a potential relation to the holographic theories.) In this case, we do not expect the color superconductor to be the correct ground state of the cold QCD. Heuristically, this is because the Cooper pair is not a color singlet and not expected to survive the limit. The possibility of a color singlet condensate of particle and hole (not anti-particle) has been investigated by Deryagin, Grigoriev and Rubakov (DGR) in Reference [13. When the particle and hole at the antipodal points of the Fermi sphere form a pair, the condensate is not homogeneous nor isotropic but is a standing wave in a certain direction;

$$
\left\langle\bar{q}_{L}(x) q_{R}(y)\right\rangle=e^{i \vec{p}_{F} \cdot(\vec{x}+\vec{y})} f(x-y),
$$

where $\left|\vec{p}_{F}\right|=\mu$ and $f$ is a function that describes the amplitude of the standing wave. The condensate is called the chiral density wave $(\chi \mathrm{DW})$ and it breaks the chiral symmetry but not the gauge symmetry (in the limit $x \rightarrow y$ ). This condensate is kinematically less favored than the Cooper pairing at $N_{c} \approx 3$, but it has been shown that the ground state instability due to the formation of $\chi \mathrm{DW}$ (DGR instability) dominates over the BCS-type instability in the large $N_{c}$ limit [13, 14.

The aim of this paper is to examine the high density behavior of the Sakai-Sugimoto model [15, 16]. As we will describe in Section 2, this is a model in Type IIA string theory with a certain brane configuration. What makes this model interesting is that the low

\footnotetext{
${ }^{2}$ This model with $N_{f}=3$ massless quarks is an approximate QCD where the masses of $u, d, s$ are set to zero and those of $c, b, t$ are taken to infinity.
} 
energy spectrum is similar to that of QCD, especially, there are fundamental quarks with the left- and right-chiral symmetries. In the strong coupling gravity background analysis, Sakai and Sugimoto have shown that the chiral symmetry is broken and they were able to compute the hadron spectrum of the theory. Moreover, they have constructed the low energy effective action of the theory with the Skyrme term whose soliton excitations can be considered as baryons. Those promising successes make this model an interesting candidate for holographic QCD. Therefore, we are naturally motivated to examine the model at high density and ultimately, we hope the model to give insight into the structure of the QCD phase diagram in all values of the parameters, especially in the medium density region where the theory is strongly coupled.

Though we do not get this far in this work, we show in Section 2 that the weak coupling regime of the theory at high density and zero temperature behaves very similar to QCD with a few differences. One of the differences is that when the chemical potential is very large with respect to the compactification size of the model, the BCS and DGR instabilities at $N_{c} \approx 3$ and $N_{c} \rightarrow \infty$, respectively, are absent and the ground state is the Fermi liquid. In Section 3, we comment on the known finite density analysis of the model at strong coupling, contrasting to the QCD expectations. In the strong coupling analysis, the possibilities of the superconductivity and $\chi \mathrm{DW}$ have not been addressed and we discuss some prospects in this direction.

\section{Weak Coupling Field Theory Analysis}

In this section, we discuss the Sakai-Sugimoto brane system at high density with the weak Yang-Mills coupling of the world-volume theory. Though the results are relatively straightforward, the computations are somewhat involved. We therefore split the discussion into the qualitative and quantitative parts. In the first part, we qualitatively explain the high density behavior of the system, then in the second part, we carry out the computations and confirm the qualitative expectations.

\subsection{Qualitative Discussion}

\subsubsection{BCS and DGR Instabilities}

Let us briefly review the BCS and DGR instabilities of high density QCD in a way that would provide the conceptual background for the quantitative calculations 3 For simplicity, we set all current quark masses to zero, which is a good approximation when the quark chemical potential is much larger than the mass of the heaviest quark. In the presence of the quark chemical potential, $\mu$, at zero temperature, we have a well-defined Fermi sphere of radius $\mu$. As a convention, we take the excitations near the Fermi surface be particles as opposed to anti-particles. When $\mu$ is very large, the anti-particles are buried deep in the Dirac sea and will not play a role in the following discussion.

On the Fermi surface, the free energy of the states are zero (more precisely, at the minimum) and it costs no free energy to change momentum along the Fermi surface. Therefore, the energy scales only in the radial direction of the sphere and we consider the renormalization group flow as we scale the energy down toward the Fermi surface. The relevant degrees of freedom are the particles and holes near the Fermi surface and we are interested in the

\footnotetext{
${ }^{3}$ For a modern exposition of the BCS instability, see Polchinski's TASI lecture notes [17].
} 
effective theory that describes the dynamics of those excitations. Let us specify a point on the Fermi surface by the momentum $\vec{p}_{F}$ with the magnitude $\left|\vec{p}_{F}\right|=\mu$ and decompose a four-momentum near this point as

$$
p^{\nu}=\left(E, \vec{p}_{F}+\vec{l}_{\|}+\vec{l}_{\perp}\right),
$$

where $\vec{l}_{\|}$is parallel to $\vec{p}_{F}$ and $\vec{l}_{\perp}$ is perpendicular to it. As stated before, only $l_{\|}$scales with energy and $l_{\perp}$ may be trivially integrated along the Fermi surface in a diagram computation. We, therefore, have the $1+1$-dimensional effective theory that describes the dynamics of the particles and holes 4 It is important to notice that the kinematics is restricted because the dynamics must take place near the Fermi surface. When we consider a particle-particle or hole-hole scattering, it is clear that the scattering must be near back-to-back, that is, the scattering pairs must be at antipodal points of the Fermi sphere. In the back-to-back scattering, the scattering angle may be arbitrary without spoiling the kinematic restriction and hence the phase space of this scattering is all over the Fermi surface.

Now in a two-dimensional theory, irrelevant operators of four dimensions may become relevant or marginal. In particular, a four-fermion interaction is marginal in two dimensions. If the interaction is attractive, the quartic coupling grows as we scale the energy down toward the Fermi surface and it eventually hits the Landau pole. This implies that the perturbation theory breaks down at the infrared scale around the pole. For the scattering of particle or hole pairs, this indicates that the naive ground state of the weakly interacting particles and holes near the Fermi surface, the Fermi liquid, is unstable against the formation of Cooper pairs. This is the BCS instability, and the new ground state has a gap due to the formation of the condensate whose size is roughly the location of the Landau pole. Technically, as we will see in the next subsection, the instability is closely related to the infrared divergence that appear in the perturbation theory and the gap properly provides the infrared cutoff. In weakly coupled QCD, i.e., QCD at very high density, the leading order contribution to the interaction is given by the one-gluon exchange and it is attractive in the antisymmetric $\overline{\mathbf{3}}$-channel, resulting in the color superconductivity.

We now turn to the DGR instability. This is associated with the scattering of the particle and hole located at the antipodal points of the Fermi sphere. In this case, the scattering is not back-to-back, but near forward. Unlike the back-to-back case, the scattering angle cannot be too large to stay near the Fermi surface and the phase space of the forward scattering is limited to a very tiny patch on the Fermi surface. The difference in the phase spaces for the BCS and DGR cases will be important in the quantitative computations.

Now the particle-hole forward scattering is similar to the Bhabha scattering whose amplitude has the forward enhancement. Thus also in our case, we can expect an infrared divergence (the DGR instability) as the exchange gluon becomes very soft, leading to a condensate of the particle-hole pair $(\chi \mathrm{DW})$. This, however, does not happen in weakly coupled QCD. The reason is that the finite density screening effect completely overwhelms such a condensate; it is the screening that provides the infrared cutoff and not the formation of a condensate.

The situation is different in the large $N_{c}$ limit (with small 't Hooft coupling). First, note that the Cooper pair of the BCS instability is not color singlet while the $\chi \mathrm{DW}$ of DGR is. Therefore, the BCS instability is $1 / N_{c}$ suppressed and DGR is not. Secondly,

${ }^{4}$ A rigorous derivation of the high density effective theory of QCD was carried out by Hong [18]. 
since only the quarks are charged under the $U(1)_{V}$-symmetry, the finite density screening effect is provided by the quark loops, such as the one shown in Figure 1, and the gluon loops do not contribute because the gluon propagator is independent of the quark chemical potential. This implies that the finite density screening is suppressed as the number of the

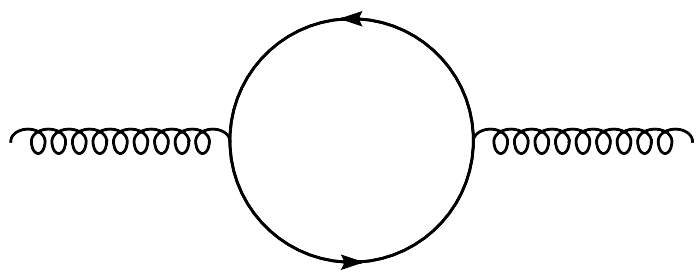

Figure 1: One loop diagram that contributes to the finite density screening.

color is taken to a large value. In fact, the DGR instability was discovered by disregarding the the screening effect and the authors of Reference [13] noted that the $\chi \mathrm{DW}$ can form and dominate over the Cooper pairing at least in the large $N_{c}$ limit. Later, Shuster and Son showed that the DGR instability may occur if $N_{c} \gtrsim 1000 N_{f}$, where $N_{f}$ is the number of the flavor [14].

This is an example where the large $N_{c}$ limit of QCD yields qualitatively different properties. In weakly coupled QCD, $\chi \mathrm{DW}$ is not a relevant phenomenon. Though it could possibly compete with the Cooper pair at strong coupling, so far the situation is unclear 19 .

\subsubsection{Sakai-Sugimoto Brane System at High Density}

We now consider whether the Sakai-Sugimoto model at high density exhibits similar properties as discussed above. For this purpose, we assume that the model is in the regime with low energy and weak Yang-Mills coupling so that we can use the perturbative world-volume field theory arguments.

The model is Type IIA string theory with D4-, D8- and $\overline{\mathrm{D} 8}$-branes. The configuration of the branes is shown in Figure 2. The $x_{4}$-direction is compactified to the circle of circumference $L$ and the $\mathrm{D} 8 \overline{\mathrm{D} 8}$-branes are placed at the antipodal points of the circle. To discuss the low energy spectrum of the model, we first consider only the compactified $N_{c}$ D4-branes. In Reference [20], Witten suggested that if we impose the anti-periodic boundary condition to the adjoint world-volume fermions, the low energy world-volume theory has the spectrum of $3+1$-dimensional pure Yang-Mills theory. This is because the fermions get tree level mass of order $1 / L$ and the scalars, including the compactified component of gauge field, $A_{4}$, get the one-loop mass of order $g / L$, where $g:=g_{5} / \sqrt{L}$ and $g_{5}^{2}:=(2 \pi)^{2} g_{s} l_{s}$, with $g_{s}$ string coupling and $l_{s}$ string length scale, and we assume $g \ll 15$

Now, Sakai and Sugimoto insert the $N_{f} \mathrm{D} 8$ and $N_{f} \overline{\mathrm{D} 8}$ branes as shown. As explained in Reference [21], there are massless fermions at the $3+1$-dimensional intersection of D4and D8-branes. These are the lowest states of the strings stretching from D8 to D4. Since the world-volume $U\left(N_{f}\right)_{L}$ gauge symmetry of D8-branes acts as the flavor symmetry, these massless fermions are fundamental "quarks". Similar massless fermions are also present

\footnotetext{
${ }^{5}$ Unlike four-dimensional case, other components of the gauge field do not acquire mass of order $g^{2} / L$, in all orders of the perturbation theory.
} 


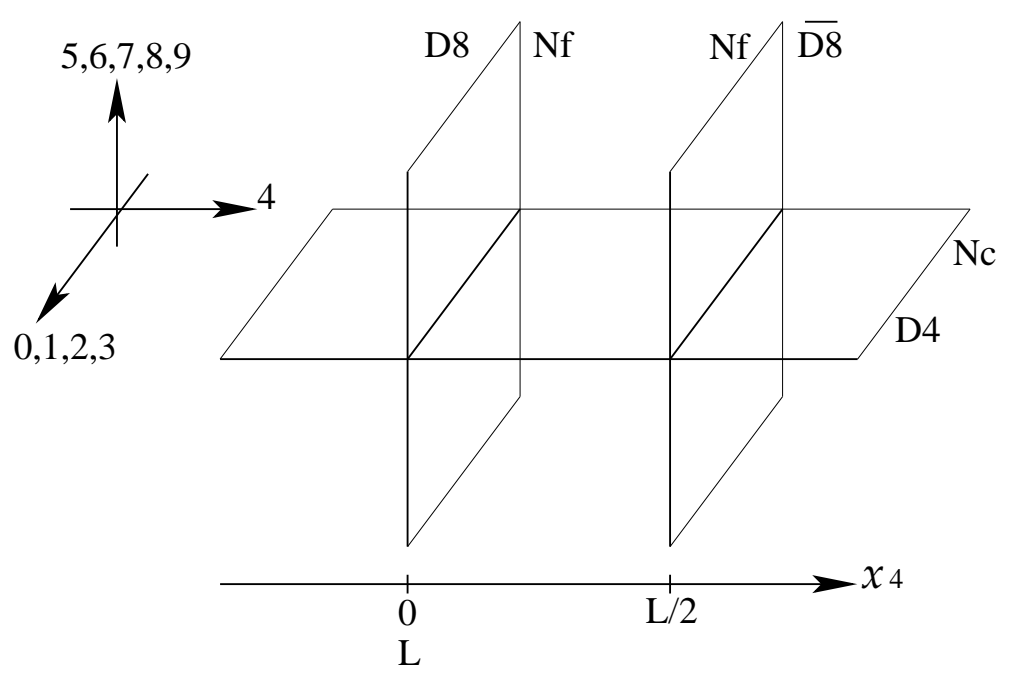

Figure 2: Sakai-Sugimoto brane configuration. The $x_{4}$-direction is compactified with period $L$. The D4 world-volume fermions have the anti-periodic boundary condition. We locate the $N_{f}$ D8-branes and $N_{f}$ $\overline{\mathrm{D}} 8$-branes at $x_{4}=0=L$ and $x_{4}=L / 2$, respectively.

at the intersection of D4- and $\overline{\mathrm{D} 8}$-branes. However, the GSO projection projects out opposite chiralities to those fermions at different intersections. Therefore, we call the massless fermions at D4D8 and D4 $\overline{\mathrm{D} 8}$ intersections as "left-handed $\left(q_{L}\right)$ and right-handed $\left(q_{R}\right)$ quarks", respectively.

This theory at low energy, therefore, has $U\left(N_{f}\right)_{L} \times U\left(N_{f}\right)_{R}$ flavor symmetry and the theory appears to be very similar to the massless QCD, if $N_{c}$ and $N_{f}$ are appropriately chosen. The difference, of course, is that the fermions with different chiralities are separately located in the $x_{4}$-direction and the gluons propagate in five dimensions, including the $x_{4^{-}}$ direction. As argued by Antonyan et al. 222, this QCD-like dynamics of the quarks at the intersections does not change even if the period $L$ is large; the shift symmetry of the D4 adjoint scalars and the five dimensional gauge symmetry allow the scalars, including $A_{4}$, to couple to the fundamental fermions only through derivative interactins which is suppressed by the string scale.

In the weak coupling analysis that we carry out in this section, we assume that the period $L$ is much larger than the string length scale $l_{s}$ so that the tachyon becomes heavy and decouples 6 Also as we have already explained, we place the $\mathrm{D} 8$ and $\overline{\mathrm{D} 8}$ branes at the antipodal points of the compactified circle.

In examining this model, we need to decide on how the quarks interact through the exchange of the gluons that propagate in the $x_{4}$-direction. One possibility is the non-local interaction. This scenario takes only into account of the zero-mode of the discrete momentum in the $x_{4}$-direction. In this case, the theory becomes completely insensitive to the existence of the fifth dimension and behaves in the same way as the four dimensional

\footnotetext{
${ }^{6}$ When the D4-branes of the system are replaced with their effective geometry (which is not the case in our discussion), the proper distance of the D8s becomes less than the string scale in the region sufficiently near the horizon and the statement made here is no longer valid in the analysis with the background geometry. Refs. 23, 24, 25] show that the tachyon indeed condenses and it is responsible for the chiral symmetry breaking of the model at strong coupling.
} 
QCD-like theory. We find this non-local scenario less appealing, especially when the compactification scale $L$ is large. We thus take the second alternative where the D8-branes are treated as sources (or stiff walls) and allow the exchanged gluons to carry arbitrarily high momenta in the $x_{4}$-direction 7 Such an assumption is reasonable because the $\mathrm{D} 8$ branes are infinitely heavier than the D4s and consistent with the fact that we are treating the D8s as the flavor branes, that is, we are neglecting their fluctuations. The momentum is not conserved in the $x_{4}$-direction but this is natural in that the translation symmetry is broken in this direction.

We now explain how we introduce the quark chemical potential. We have the global symmetry $S U\left(N_{f}\right)_{L} \times S U\left(N_{f}\right)_{R} \times U(1)_{V}$. The chemical potential that we are interested in is conjugate to the $U(1)_{V}$ charge. The standard way to introduce a chemical potential in a field theory is to treat it as the constant background "gauged" field of a $U(1)$ global symmetry, with all the components being zero except the time component. In this way, the chemical potential modifies the time component of the covariant derivatives in the Lagrangian of the theory. Thus in our case, the simplest way to introduce the quark chemical potential is to turn on the $A_{0}$ constant background gauge fields of $U(1) \in U\left(N_{f}\right)_{L}$ and $U(1) \in U\left(N_{f}\right)_{R}$ world-volume gauge symmetries and tune them to an equal value. Actually, the background fields may not be constant all over the D8-branes and the only requirement is to have the constant value at the intersections with the D4-branes. Thus, for example, we may turn on the field that depends on the radial direction in the 5,6,7,8 and 9 directions which would correspond to the nonzero electric field in the world-volume.

What we will find in the quantitative analysis of the next section is rather intuitive. As we have mentioned, if we consider the effect only of the zero-mode momentum in the $x_{4}$-direction, the theory reduces to the QCD-like theory. Thus when the compactification scale $1 / L$ is very large compared to the energy scale of the interest, in this case it is the value of the chemical potential $\mu$, we expect to have the BCS and DGR instabilities at $N_{c} \approx 3$ and at $N_{c} \rightarrow \infty$, respectively, just as described before. Now as the compactification scale $1 / L$ gets smaller, the infrared effect in the $x_{4}$-direction becomes comparable to the one that leads to the regular BCS or DGR instability. As a consequence, the size of the condensate grows and eventually becomes too large to maintain the dynamics near the Fermi surface. Therefore, when the scale $1 / L$ is small with respect to $\mu$, there is no BCS or DGR type of instability and the ground state of the theory is described by the Fermi liquid.

In this qualitative discussion, it is not clear at what scale this crossover occurs. The computations of the next subsection show that at $N_{c} \approx 3$ and $\mu L \gtrsim 1 / g$, no BCS-type instability is present and at $N_{c} \rightarrow \infty$ and $\mu L \gtrsim e^{1 / \sqrt{\lambda}} / \sqrt{\lambda}$ with $\lambda:=g^{2} N_{c}$, no DGR-type instability happens. Notice that the DGR instability persists to exponentially larger value of $\mu L$ compared to the BCS case. This is because the phase space of the particle-hole scattering is very small, in fact it is exponentially small, and the discrete momentum in the compactified direction must become as fine as this scale to open up the extra dimension. The situation explained here is schematically summarized in Figure 3 ,

\footnotetext{
${ }^{7}$ The gluon momenta actually have to be cut off below the string scale to avoid the derivative interactions between the D4 adjoint scalars and the fundamental fermions. However, as we will see shortly, the high momenta increasingly suppress the gluon propagator and their contributions become negligible at sufficiently high scale. Therefore we can approximately take the $x_{4}$-momentum to infinity.
} 


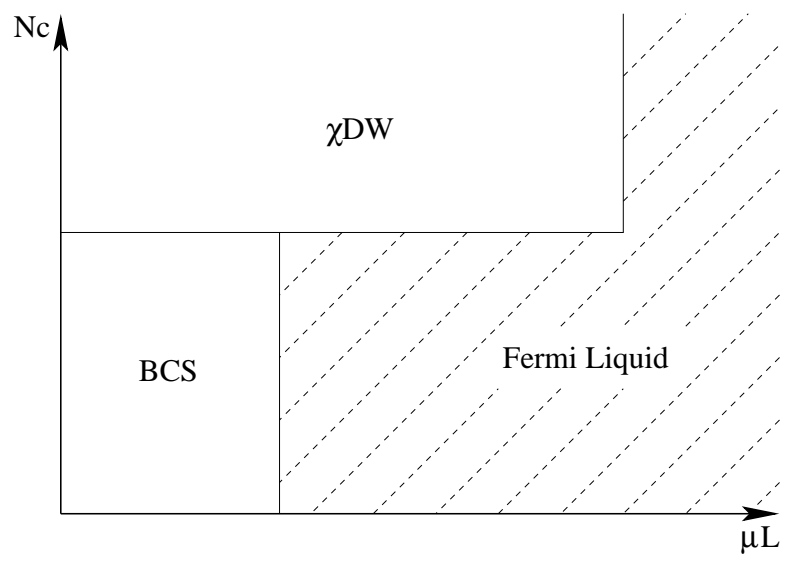

Figure 3: A schematic phase diagram of the theory at weak coupling, high density and zero temperature. Being schematic, the straight lines may not be straight nor sharp transitions in reality.

\subsection{Quantitative Discussion}

We now demonstrate quantitatively what has been discussed in the previous subsection. We carry out the renormalization group and Dyson-Schwinger analyses. The former is more intuitive in accordance with the qualitative discussion and shows the existence of the instabilities. But this method does not provide the size of the gap and this is augmented by solving the Dyson-Schwinger equations.

We adopt the conventions of Wess and Bagger [26], except the definition of the Dirac spinor;

$$
\psi:=\left(\begin{array}{c}
q_{L \alpha} \\
q_{R}^{\dot{\alpha}}
\end{array}\right), \quad \bar{\psi}:=\left(\bar{q}_{R}{ }^{\alpha}, \bar{q}_{L \dot{\alpha}}\right)
$$

We mainly work in the chiral basis. As a convention, the undotted and dotted spinors live on the $\mathrm{D} 8$ and $\overline{\mathrm{D}} 8$ branes, respectively.

Our central focus of this subsection is to show the existence of the instabilities and to obtain the size of the gap. We are less interested in the exact color-flavor structure of the condensate, so in what follows, we simplify the analysis by suppressing the flavor structure. This is similar to $N_{f}=2$ case where the Pauli principle requires the simpler quark pairing.

\subsubsection{Renormalization Group Equations}

Our first analysis is macroscopic in a sense that we introduce an effective one point fourfermion coupling. Then we observe how the effective coupling evolves as we scale the energy of the system down to the Fermi surface. This idea was first carried out in high density QCD by Evans et al. in Reference [27.

Since we are dealing with the weak coupling at high density, the quark interaction can be approximated by a single gluon exchange. We then model the four-fermion interaction by replacing the one-gluon exchange to a point, as shown in Figure 4. Because the chemical potential breaks the $3+1$ world-volume symmetry down to $O(3)$, we separately handle the couplings, $G^{0}$ and $G^{j}$, as in

$$
i G^{0}\left(\bar{\psi} \gamma^{0} \psi\right)^{2}, \quad i G^{j}\left(\bar{\psi} \gamma^{j} \psi\right)^{2}
$$



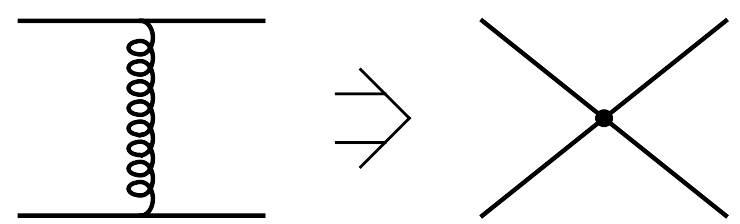

Figure 4: Replacing the one-gluon exchange to an effective one point interaction.

For the one-gluon exchange, we can further write

$$
G^{0}(D)=-G^{j}(D):=-g_{5}^{2} X(D) F .
$$

Notice that we have included the minus sign from the signature in the definition of $G^{0}$. The constant $g_{5}$ is the five dimensional Yang-Mills coupling as before, $F$ is the form factor that arises from the gluon propagator and

$$
X(D):=\frac{1}{2}\{C(D)-2 C(\square)\}
$$

with $C(D)$ being the Casimir operator of $S U\left(N_{c}\right)$ in the representation $D$ and $\square$ being the defining representation.

We consider three color channels; symmetric (symm), antisymmetric (asymm) and singlet $(\bullet)$. For those cases, we have

$$
X(\operatorname{symm})=\frac{N_{c}-1}{2 N_{c}}, \quad X(\operatorname{asymm})=-\frac{N_{c}+1}{2 N_{c}}, \quad X(\bullet)=-\frac{N_{c}^{2}-1}{2 N_{c}},
$$

where we have adopted the normalization $\operatorname{tr}\left(T^{\alpha} T^{\beta}\right)=(1 / 2) \delta^{\alpha \beta}$ for the $N_{c} \times N_{c}$ matrices $\left\{T^{\alpha}: \alpha=1, \ldots, N_{c}^{2}-1\right\}$ of the defining representation. Notice the large $N_{c}$ behavior of those factors. The singlet channel is larger than the other channels by a factor of $N_{c}$ in the absolute value. This can be easily understood in the double line notation of the single gluon exchange diagrams, as shown in Figure 5.
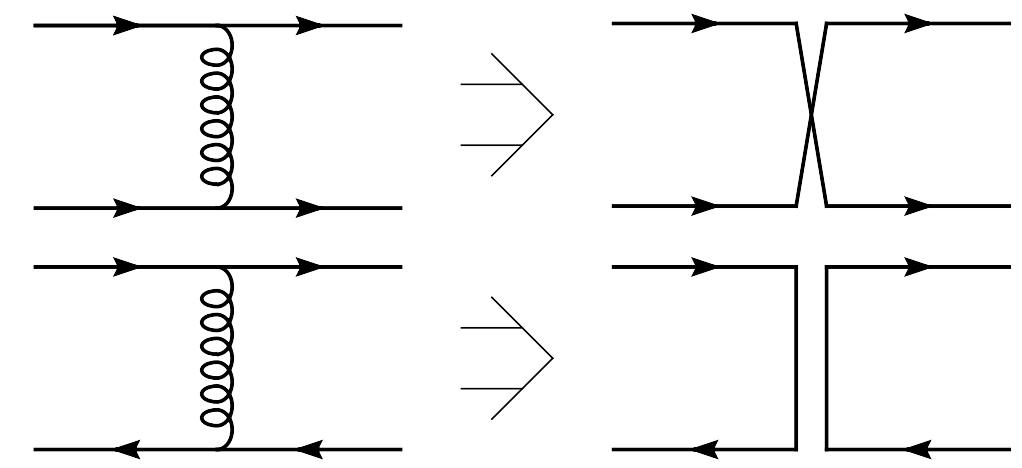

Figure 5: The double line notations of the one-gluon exchange. The top and bottom sets represent the symmetric (or antisymmetric) and singlet channels, respectively. If we fix the colors of the incoming quarks, the symmetric channel has the fixed colors for the scattered quarks, while the singlet channel has $N_{c}$ choices.

Let us now consider the form factor, $F$. The gluon propagator in the Feynman gauge has the form $1 /\left(p^{2}+p_{4}^{2}\right)$ with the discrete momentum, $p_{4}=2 \pi n / L$, in the compactified direction and this propagator suffers from an infrared divergence. To cure this problem, we introduce 
an infrared cutoff $m$. We simplify the situation by assuming that $m$ is the same for the time and spatial components of the gluons 8 Possible one-gluon interactions are illustrated in Figure 6. In both cases shown in the figure, the discrete momentum is $2 \pi n / L$ because this
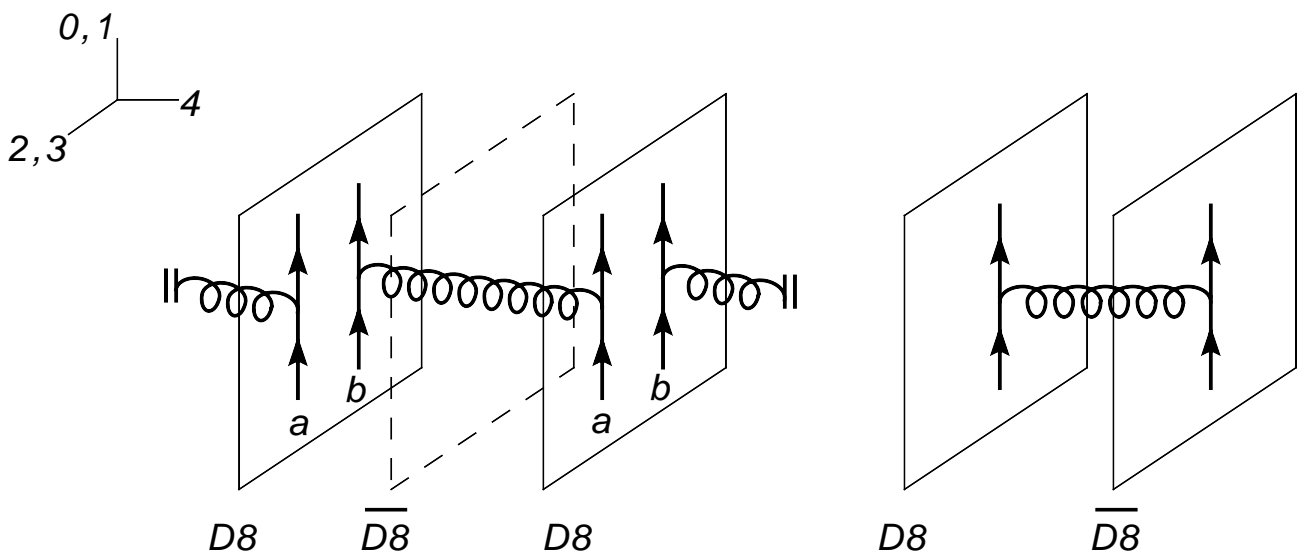

Figure 6: One-gluon exchange diagram. The gluons are propagating in the compactified $x_{4}$-direction. The D8 $\overline{\mathrm{D} 8}$-branes are treated as sources or stiff walls.

is determined by the period of the compactification and not by the distance between the branes. Therefore, the form factor, that arises from the gluon propagator, is independent of the D8 brane distance and is the same for the both interactions in Figure 6 (including the case with the gluons making many rounds in the compactified circle). We follow Evans et al. [27] to obtain the form factor, namely, we take average of the gluon propagator over the scattering phase space. For the dynamics very near the Fermi surface, the energy and the component of the momentum in the radial direction of the Fermi sphere are almost zero. Hence apart from the extra $x_{4}$-direction, the phase space is two dimensional along the Fermi surface. Let $p_{1}$ and $p_{2}$ be the incoming and outgoing momenta of a scattering quark. We then have the gluon momentum

$$
p^{2}=\left(p_{1}-p_{2}\right)^{2} \approx 2 \mu^{2}(1-\cos \theta),
$$

where $\theta$ is the scattering angle.

For the back-to-back scattering, the angle ranges from 0 to $\pi$ and the phase space is all over the Fermi surface. Therefore, the form factor in this case is

$$
\begin{aligned}
F_{B B} & =\frac{1}{\mathcal{N} L} \sum_{n=-\infty}^{\infty} \int \frac{d^{2} p}{p^{2}+(2 \pi n / L)^{2}+m^{2}} \\
& =\frac{2 \pi}{\mathcal{N} L} \ln \left[\sinh \left(\frac{1}{2} m L \sqrt{1+4 \mu^{2} / m^{2}}\right) / \sinh \left(\frac{1}{2} m L\right)\right],
\end{aligned}
$$

where we have defined the total phase space factor $\mathcal{N}:=4 \pi \mu^{2}$. We have already carried out the sum over the $p_{4}$-discrete momentum because the $p_{4}$ dependence is only in the gluon

\footnotetext{
${ }^{8}$ At very high density where the coupling is weak, one can expect the finite density Debye screening of order $g \mu$ provides the infrared cutoff. This is true for the time component but not for the spatial components. There is no static screening in the spatial (magnetic) components and the magnetic screening is dynamical due to the Landau damping. This was pointed out by Son 28] and he discovered that the dynamical screening effect leads to the qualitatively different form of the gap. We will take into account of Son's effect in the Dyson-Schwinger analysis.
} 
propagator. As an effect, we have encoded all the information about the extra dimension into the form factor. For the forward scattering of a particle-hole pair, the angle should not take very large value so that the dynamics takes place near the Fermi surface. Thus we require, in this case, the angle range from 0 to $\theta_{U V}$, where the latter angle is much less than $\pi$ and limits the phase space to a little patch on the Fermi surface. The form factor then takes the form

$$
\begin{aligned}
F_{F W} & =\frac{1}{\mathcal{M} L} \sum_{n=-\infty}^{\infty} \int \frac{d^{2} p}{p^{2}+(2 \pi n / L)^{2}+m^{2}} \\
& =\frac{2 \pi}{\mathcal{M} L} \ln \left[\sinh \left(\frac{1}{2} m L \sqrt{1+2\left(1-\cos \theta_{U V}\right) \mu^{2} / m^{2}}\right) / \sinh \left(\frac{1}{2} m L\right)\right],
\end{aligned}
$$

where $\mathcal{M}:=2 \pi \mu^{2}\left(1-\cos \theta_{U V}\right)$. Notice that since $\theta_{U V} \ll \pi$, we have $\mathcal{M} F_{F W}<\mathcal{N} F_{B B}$. This fact will lead to the dominance of the BCS-type instability over the DGR type for $N_{c} \approx 3$. When the dimensionless parameter $m L$ is small, the form factors behave logarithmically with respect to the parameter $\mu / m$ and they behave linearly when $m L$ is large. This is because when the the compactified dimension is very small, the contributions from $n \neq 0$ is also small and the integral is effectively two dimensional, leading to the log behavior. When the compactification size is very large, the discrete momentum becomes finer and the integral essentially becomes three dimensional and the form factors behave linearly.

Having modeled the four-fermion interaction, we now derive the renormalization group equations for the couplings. The diagram that drives the renormalization group flow is the fermion one-loop diagram and we consider the three cases shown in Figure 7 , For Diagram

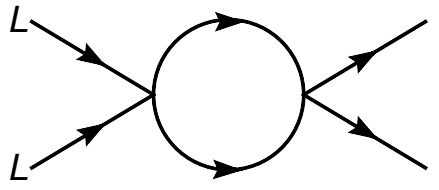

(a)

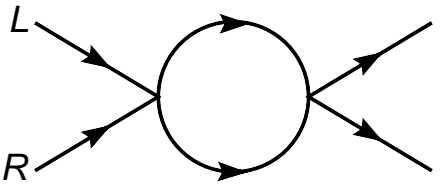

(b)

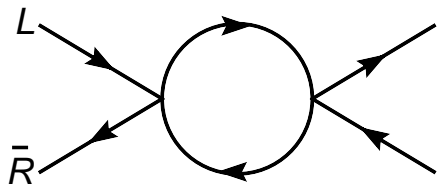

(c)

Figure 7: The diagrams that drive the renormalization group flow. The letters " $L$ " and " $R$ " refer to leftand right-handed quarks, respectively.

(a) in the back-to-back scattering, we can deduce from the expressions (6) that each vertex corresponds to either

$$
i G_{L L}^{0} \bar{\sigma}^{0 \dot{\alpha} \alpha} \bar{\sigma}^{0 \dot{\beta} \beta}, \quad \text { or } \quad i G_{L L}^{j} \bar{\sigma}^{j \dot{\alpha} \alpha} \bar{\sigma}^{j \dot{\beta} \beta} .
$$

When the both vertices correspond to $G_{L L}^{0}$, the diagram yields

$$
\left(i G_{L L}^{0}\right)^{2}\left(\bar{\sigma}^{0 \dot{\delta} \alpha} \bar{\sigma}^{0 \dot{\gamma} \beta}\right) \int \frac{d^{4} p}{(2 \pi)^{4}}\left[\frac{-i\left(p_{\nu}-\mu \delta_{\nu, 0}\right) \sigma_{\alpha \dot{\alpha}}^{\nu}}{\left(p_{\lambda}-\mu \delta_{\lambda, 0}\right)^{2}} \frac{-i\left(-p_{\eta}-\mu \delta_{\eta, 0}\right) \sigma_{\beta \dot{\beta}}^{\eta}}{\left(-p_{\lambda}-\mu \delta_{\lambda, 0}\right)^{2}}\right]\left(\bar{\sigma}^{0 \dot{\alpha} \delta} \bar{\sigma}^{0 \dot{\beta} \gamma}\right)
$$

where we used the quark propagators shown in Appendix $\mathrm{A}$. Note that the momentum integral is four dimensional rather than five. The dynamics of the quarks are restricted to the four dimensional intersections of the branes and the information about the extra dimension has been encoded in the form factor. We decompose the momentum as in Equation (4) but redefine $\vec{p}_{F}$ to include $\vec{l}_{\perp}$. Then near the surface of the large Fermi sphere, the 
vectors $\vec{p}_{F}$ and $\vec{l}_{\|}$are near parallel and we also have $E, l_{\|} \ll \mu$ and $p_{F} \approx \mu$. Under these approximations together with the use of the $O(3)$-invariance, the argument of the square bracket in Equation (14) becomes

$$
-\frac{1}{4}\left(-\sigma_{\alpha \dot{\alpha}}^{0} \sigma_{\beta \dot{\beta}}^{0}+\frac{1}{3} \sigma^{j}{ }_{\alpha \dot{\alpha}} \sigma_{\beta \dot{\beta}}^{j}\right) \frac{1}{E^{2}-l_{\|}^{2}} .
$$

One can integrate over $E$, either by the contour integral or by the Wick rotation, then $\left|l_{\|}\right|$ is integrated from the scale $\Lambda_{U V}$ down to $\Lambda_{I R}$. One can also simplify the $\sigma$-matrices (all the necessary formulas are given in Appendix B of Wess and Bagger [26]) and the expression (14) becomes

$$
\frac{i \mathcal{N}}{16 \pi^{3}}\left(G_{L L}^{0}\right)^{2}\left(\bar{\sigma}^{0 \dot{\delta} \delta} \bar{\sigma}^{0 \dot{\gamma} \gamma}-\frac{1}{3} \bar{\sigma}^{j \dot{\delta} \delta} \bar{\sigma}^{j \dot{\gamma} \gamma}\right) t,
$$

where we have defined $t:=\ln \left(\Lambda_{I R} / \Lambda_{U V}\right)$. This parameter $t$ has the range $(-\infty, 0)$ and the lower limit corresponds to the Fermi surface. When one of the vertex of Diagram (a) is $G^{0}$ and the other is $G^{j}$, similar procedure yields

$$
\frac{i \mathcal{N}}{16 \pi^{3}}\left(G_{L L}^{0} G_{L L}^{j}\right)\left(-2 \bar{\sigma}^{0 \dot{\delta} \delta} \bar{\sigma}^{0 \dot{\gamma} \gamma}+\frac{10}{3} \bar{\sigma}^{j \dot{\delta} \delta} \bar{\sigma}^{j \dot{\gamma} \gamma}\right) t
$$

and when the vertices are both $G^{j}$, we get

$$
\frac{i \mathcal{N}}{16 \pi^{3}}\left(G_{L L}^{j}\right)^{2}\left(5 \bar{\sigma}^{0 \dot{\delta} \delta} \bar{\sigma}^{0 \dot{\gamma} \gamma}-\frac{13}{3} \bar{\sigma}^{j \dot{\delta} \delta} \bar{\sigma}^{j \dot{\gamma} \gamma}\right) t .
$$

From those results, we obtain the renormalization group equations

$$
\begin{aligned}
& \frac{d G_{L L}^{0}}{d t}=\frac{\mathcal{N}}{16 \pi^{3}}\left\{-\left(G_{L L}^{0}\right)^{2}+2 G_{L L}^{0} G_{L L}^{j}-5\left(G_{L L}^{j}\right)^{2}\right\}, \\
& \frac{d G_{L L}^{j}}{d t}=\frac{\mathcal{N}}{16 \pi^{3}}\left\{\frac{1}{3}\left(G_{L L}^{0}\right)^{2}-\frac{10}{3} G_{L L}^{0} G_{L L}^{j}+\frac{13}{3}\left(G_{L L}^{j}\right)^{2}\right\} .
\end{aligned}
$$

These equations can be diagonalized to the following forms

$$
\begin{aligned}
\frac{d\left(G_{L L}^{0}-3 G_{L L}^{j}\right)}{d t} & =-\frac{\mathcal{N}}{16 \pi^{3}}\left(G_{L L}^{0}-3 G_{L L}^{j}\right)^{2}, \\
\frac{d\left(G_{L L}^{0}+G_{L L}^{j}\right)}{d t} & =-\frac{\mathcal{N}}{48 \pi^{3}}\left(G_{L L}^{0}+G_{L L}^{j}\right)^{2} .
\end{aligned}
$$

One can carry out the same procedure for the back-to-back scattering of Diagram (b) in Figure 7 and obtain

$$
\begin{aligned}
\frac{d\left(G_{L R}^{0}+3 G_{L R}^{j}\right)}{d t} & =0 \\
\frac{d\left(G_{L R}^{0}-G_{L R}^{j}\right)}{d t} & =-\frac{\mathcal{N}}{24 \pi^{3}}\left(G_{L \bar{R}}^{0}-G_{L \bar{R}}^{j}\right)^{2} .
\end{aligned}
$$

Above four renormalization group equations are obtained by Evans et al. [27] (but with different form factors). 
The Diagram (c) of Figure 7 is similar to the case with (a) [and not with (b) because $\bar{q}_{R}$ has an undotted spinor index just as $\left.q_{L}\right]$. But we should recall that we are interested in the forward scattering in this case. Therefore in the loop, the top left-handed quark propagator carries the momentum $\left(E, \vec{p}_{F}+\vec{l}_{\|}\right)$and the bottom right-handed one carries $\left(E,-\vec{p}_{F}+\vec{l}_{\|}\right)$ in the directions of the arrows in the quark loop. Then the propagator part of the diagram corresponding to (15) of the Diagram (a) is

$$
-\frac{1}{4}\left(\sigma^{0}{ }_{\alpha \dot{\alpha}} \bar{\sigma}^{0 \dot{\beta} \beta}-\frac{1}{3} \sigma^{j}{ }_{\alpha \dot{\alpha}} \bar{\sigma}^{j \dot{\beta} \beta}\right) \frac{1}{E^{2}-l_{\|}^{2}} .
$$

This structure is the same as the $L L$-case (a), except the overall sign. This sign is cancelled by the other one that comes from the difference in the direction of the quark line. Thus, apart from the integration range of the scattering angle $\theta$, the cases (a) and (c) are the same. So we have for Diagram (c),

$$
\begin{aligned}
\frac{d\left(G_{L \bar{R}}^{0}-3 G_{L \bar{R}}^{j}\right)}{d t} & =-\frac{\mathcal{M}}{16 \pi^{3}}\left(G_{L \bar{R}}^{0}-3 G_{L \bar{R}}^{j}\right)^{2}, \\
\frac{d\left(G_{L \bar{R}}^{0}+G_{L \bar{R}}^{j}\right)}{d t} & =-\frac{\mathcal{M}}{48 \pi^{3}}\left(G_{L \bar{R}}^{0}+G_{L \bar{R}}^{j}\right)^{2} .
\end{aligned}
$$

Now for the generic form of the renormalization group equation

$$
\frac{d G(t)}{d t}=-K G(t)^{2}
$$

with some constant $K$, the Landau pole, if exists, is reached at

$$
t_{L P}=-\frac{1}{K G(0)}
$$

Since the range of $t$ is $(-\infty, 0)$, the Landau pole exists only when $K G(0)$ is positive and the larger the factor $K G(0)$ is, the faster the pole is reached. We note that because of the constants $\mathcal{N}$ and $\mathcal{M}$ in the form factors (11) and (12), the Landau pole is independent of those. From Equations (7) and (9), and also with the fact that $\mathcal{M} F_{F W}<\mathcal{N} F_{B B}$, we see that the instability is dominated by the BCS type in the $L L$, color antisymmetric channel for $N_{c} \approx 3$. Note that the color symmetric channel does not have instability because the interaction in this channel is repulsive. When $N_{c}$ is sufficiently large, the $L \bar{R}$ channel, whose coupling is proportional to the product of $X(\bullet)$ and $F_{F W}$, dominates over the other channels because the inequality $\mathcal{M} F_{F W}<\mathcal{N} F_{B B}$ is compensated by the fact that $\mid X($ asymm $)|<| X(\bullet) \mid$. Thus the DGR-type instability dominates over BCS in this regime. In our method here, it is not possible to estimate the value of $N_{c}$ at which the crossover from the BCS- to DGR-type instability occurs, because we crudely introduced the common infrared cutoff, $m$, to all the components of the gluon propagator and we do not have the actual value of $\theta_{U V}$. See Shuster and Son [14] for the estimate of the value $N_{c}$.

Recall that the form factors grow linearly when the parameters $m L$ and $\mu / m$ are large and the effective four-fermion couplings become large accordingly. We thus expect that the whole analysis breaks down when those parameters are exceedingly large, and we need to resort to the microscopic analysis, i.e., the analysis with the fundamental interactions, to gain insight into the nature of the pathology. This is the subject of the next analysis. 


\subsubsection{Dyson-Schwinger Equations}

We now turn to the analysis based on the Dyson-Schwinger equations. The traditional form of the Dyson-Schwinger equation in the diagrammatic representation is shown in Figure 8 . This method is less intuitive compared to the previous renormalization group analysis, but

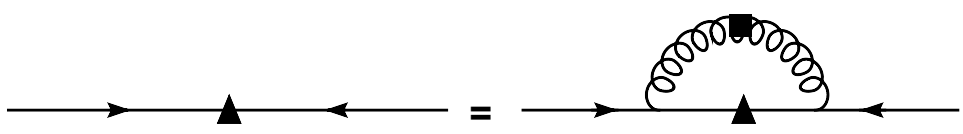

Figure 8: The diagrammatic representation of the Dyson-Schwinger equation for the diquark condensate. The triangle denotes the gap insertion. The square in the gluon propagator represents the screening effect.

it lets us obtain the actual size of the gap. This analysis is microscopic which deals with the quarks rather than the quasi-particles of the effective theory and interactions are the QCD interactions rather than the effective ones.

As usual, we adopt the Nambu-Gor'kov formalism (see, e.g., Reference [9]). In order to introduce the Nambu-Gor'kov basis, we define the charge conjugate Dirac spinors as $\psi^{C}:=$ $C \bar{\psi}^{T}$ and $\bar{\psi}^{C}:=\psi^{T} C^{T}$ where the charge conjugation matrix $C$ is defined in Appendix $\mathrm{A}$. In the Weyl basis, these can be expressed as

$$
\left(\begin{array}{l}
\bar{q}_{R \alpha}^{c} \\
\bar{q}_{L}^{c \dot{\alpha}}
\end{array}\right)=\left(\begin{array}{c}
\left(i \sigma^{2}{ }_{\alpha \dot{\beta}} \bar{\sigma}^{0 \dot{\beta} \gamma}\right) \bar{q}_{R \gamma} \\
\left(i \bar{\sigma}^{2 \dot{\alpha} \beta} \sigma_{\beta \dot{\gamma}}^{0}\right) \bar{q}_{L}^{\dot{\gamma}}
\end{array}\right), \quad\left(q_{L}^{c \alpha}, q_{R \dot{\alpha}}^{c}\right)=\left(q_{L}^{\gamma}\left(-i \sigma_{\gamma \dot{\beta}}^{2} \bar{\sigma}^{0 \dot{\beta} \alpha}\right), q_{R \dot{\gamma}}\left(-i \bar{\sigma}^{2 \dot{\gamma} \beta} \sigma_{\beta \dot{\alpha}}^{0}\right)\right) .
$$

Then we define the Nambu-Gor'kov basis as

$$
\Psi:=\frac{1}{\sqrt{2}}\left(q_{L \alpha}, q_{R}^{\dot{\alpha}}, \bar{q}_{R \beta}^{c}, \bar{q}_{L}^{c \dot{\beta}}\right)^{T}, \quad \bar{\Psi}:=\frac{1}{\sqrt{2}}\left(\bar{q}_{R}{ }^{\alpha}, \bar{q}_{L \dot{\alpha}}, q_{L}^{c \beta}, q_{R \dot{\beta}}^{c}\right) .
$$

The advantage of the Nambu-Gor'kov formalism is that we can naturally include the condensates in the propagator of $\Psi$. For example, the diquark condensate in the $s$-wave $(L L$ or $R R$ condensate) is given as

$$
\psi^{T} C^{T} \gamma^{5} \psi=-i q_{L}^{c} q_{L}+i q_{R}^{c} q_{R}
$$

thus the inverse propagator that contains this condensate can be written as

$$
G(p)^{-1}=-i\left(\begin{array}{cccc}
0 & \left(p_{\nu}-\mu \delta_{\nu, 0}\right) \sigma^{\nu} & i \bar{\Delta}_{R}(p) & 0 \\
\left(p_{\nu}-\mu \delta_{\nu, 0}\right) \bar{\sigma}^{\nu} & 0 & 0 & -i \bar{\Delta}_{L}(p) \\
i \Delta_{L}(p) & 0 & 0 & \left(p_{\nu}+\mu \delta_{\nu, 0}\right) \sigma^{\nu} \\
0 & -i \Delta_{R}(p) & \left(p_{\nu}+\mu \delta_{\nu, 0}\right) \bar{\sigma}^{\nu} & 0
\end{array}\right)
$$

The $\Delta$-matrices appearing in the inverse propagator are defined as

$$
\Delta_{L, R}(p)=\Delta_{+}(p) P_{L, R+}(p)+\Delta_{-}(p) P_{L, R-}(p),
$$

where $\Delta_{ \pm}(p)$ are the gaps and the quark (anti-quark) on-shell projectors, $P_{L, R \pm}(p)$, are defined in Appendix $\mathrm{A}$. The ones with the bar can be obtained by replacing $P \rightarrow \bar{P}$ in the above expression. (We assume the gaps to be real.) We can invert the matrix (32) by using the formulas listed in Appendix $\mathrm{A}^{9}$ If we write

$$
G(p)=\left(\begin{array}{ll}
G_{11} & G_{12} \\
G_{21} & G_{22}
\end{array}\right)
$$

\footnotetext{
${ }^{9}$ Note that the projectors are not invertible, so one must use appropriate inversion formulas.
} 
we then have

$$
G_{21}=\left(\begin{array}{cc}
-\frac{\Delta_{+} P_{L-}}{p_{0}^{2}-(|\vec{p}|-\mu)^{2}-\Delta_{+}^{2}}-\frac{\Delta_{-} P_{L+}}{p_{0}^{2}-(|\vec{p}|+\mu)^{2}-\Delta_{-}^{2}} & 0 \\
0 & \frac{\Delta_{+} P_{R-}}{p_{0}^{2}-(|\vec{p}|-\mu)^{2}-\Delta_{+}^{2}}+\frac{\Delta_{-} P_{R+}}{p_{0}^{2}-(|\vec{p}|+\mu)^{2}-\Delta_{-}^{2}}
\end{array}\right),
$$

and other components will not be important in writing down the Dyson-Schwinger equations. Notice that if the condensates, $\Delta_{ \pm}$, in the denominators vanish there are terms that diverge as the energy is scaled toward the Fermi surface, i.e., $p_{0} \rightarrow 0$ and $|\vec{p}| \rightarrow \mu$. This is essentially due to the sign structure of the chemical potential in the matrix (32) and to the fact that the $\Delta$-matrices occupy off block-diagonal components. This infrared divergence is cured by the formation of the condensate and the $\Delta$ properly behaves as such a condensate.

If this were the traditional four-dimensional set up, we could have introduced the $p$-wave diquark condensate ( $L R$-pair) in the Nambu-Gor'kov propagator. [Such condensate would have occupied the anti-diagonal slots of the matrix (32).] However, this is not allowed in our theory. As shown in Figure 9, since $q_{L}$ and $q_{R}$ separately live on the D8 and $\overline{\mathrm{D} 8}$ branes,
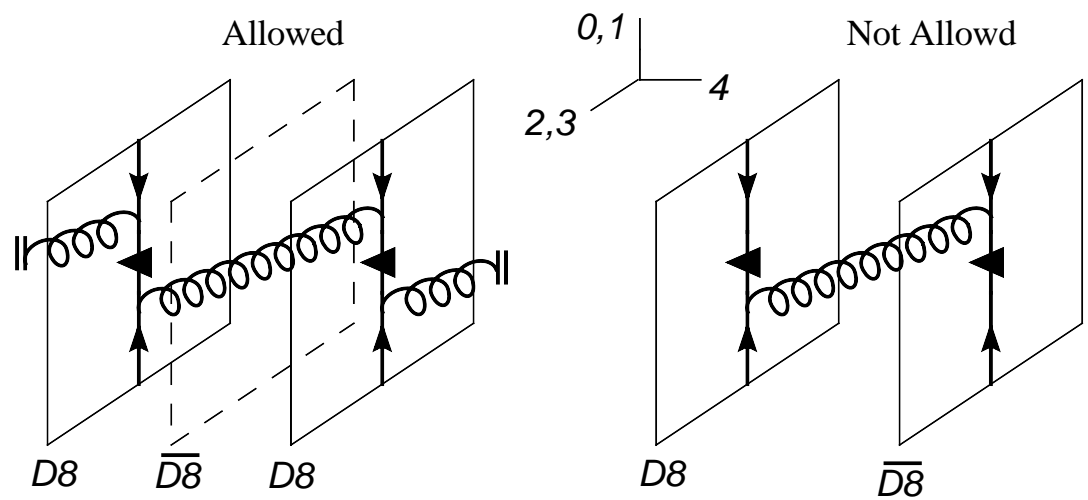

Figure 9: The left diagram represents the right-hand side of the Dyson-Schwinger equation (Figure 8) for $L L$-diquark condensate. On the right panel is the similar diagram with $L R$-diquark condensate. This clearly is not making sense, because the condensate (the triangle) is separated and also is not being able to flip the helicity.

the Dyson-Schwinger equation for such condensate cannot make sense. In the previous macroscopic renormalization group analysis, we encoded all the information about the extra dimension in the form factors and the left- and right-handed quarks effectively lived in the same four dimensional spacetime. However, in this microscopic Dyson-Schwinger analysis, we see that it is actually not possible for the $L R$-condensate to form. We emphasize that the condensate is not being energetically suppressed but simply not possible to form in the brane system of Sakai and Sugimoto 10

For the particle-hole $(L \bar{R}$ or $\bar{L} R)$ pair, we might naively introduce the condensate in the diagonal slots of the matrix in (32). However, this represents the introduction of the usual chiral condensate, that is, the pair of particle and anti-particle, and not the desired particle-hole pair. One can verify in this case that the infrared divergence near the Fermi

${ }^{10}$ Refs. [29, 30] discuss the condensation of a gauge invariant particle-antiparticle pair across the D8 and $\overline{\mathrm{D}} 8$ branes at strong coupling. Although it is possible that this sort of condensation forms in our situation, we note that we are considering the condensation of a particle pair which is not gauge invariant and at weak coupling. Therefore, this possibility is not taken into account in our discussion here. 
surface is not present. Thus, the Nambu-Gor'kov basis is not suitable for introducing the particle-hole chiral condensate. Instead, we propose to use the doubled basis,

$$
\frac{1}{\sqrt{2}}\left(q_{L \alpha}, q_{R}^{\dot{\alpha}}, q_{L \alpha}, q_{R}^{\dot{\alpha}}\right)^{T}
$$

and consider the inverse propagator of the form (32) with the replacements, $i \Delta_{L} \rightarrow \Sigma_{L}$, $-i \Delta_{R} \rightarrow \Sigma_{R},-i \bar{\Delta}_{L} \rightarrow \bar{\Sigma}_{L}$ and $i \bar{\Delta}_{R} \rightarrow \bar{\Sigma}_{R}$, where the $\Sigma$-matrices are similarly defined as for the $\Delta$-matrices. The spinors of the second set have the chemical potentials in their kinetic terms with opposite sign from the first set. This effectively introduces the hole degrees of freedom. In this case, the propagator has the infrared divergence near the Fermi surface and the $\Sigma$-condensate provides the cutoff. Thus we properly have the interpretation that the condensate is the particle-hole pair near the Fermi surface. We note that since the condensate is formed out of the spinors with the dotted- or undotted-index pair, and not the mixed one, the condensate lives either on D8 or $\overline{\mathrm{D} 8}$ branes and not across them.

We must also consider the gluon propagator which is the other ingredient of the DysonSchwinger equation. Unlike previous macroscopic treatment, we properly take into account of the perturbatively computable screening effect. The most general form of the $O(3)$ invariant gluon propagator is

$$
D_{\mu \nu}(p, n)=\frac{P_{\mu \nu}^{T}(p)}{p^{2}+(2 \pi n / L)^{2}+G_{s}(p)}+\frac{P_{\mu \nu}^{L}(p)}{p^{2}+(2 \pi n / L)^{2}+F_{s}(p)},
$$

where $F_{s}$ and $G_{s}$ are the electric and magnetic screenings, respectively, and the projectors are defined as

$$
P_{i j}^{T}(p)=\eta_{i j}-\frac{p_{i} p_{j}}{|\vec{p}|^{2}}, P_{00}^{T}(p)=0=P_{01}^{T}(p), P_{\mu \nu}^{L}(p)=\eta_{\mu \nu}-\frac{p_{\mu} p_{\nu}}{p^{2}}-P_{\mu \nu}^{T}
$$

We have dropped the term with the gauge fixing parameter in the propagator. This term has been verified not to contribute to the gap, the solution to the Dyson-Schwinger equation, at very large chemical potential [31].

As explained in Section 2.1, the finite density screening effect is given by the diagram shown in Figure 1, at one-loop level. Since the quark loop stays on the D8 or $\overline{\mathrm{D} 8}$ branes, there is no extra dimensional effect on the screening, and the gluons with nonzero momentum in the $x_{4}$-direction, such as the case shown in Figure 9, do not have the screening. We thus have the standard expressions [32]

$$
\begin{aligned}
& F_{s}(p)=2 m_{D}^{2} \frac{p^{2}}{|\vec{p}|^{2}}\left[1-\frac{p_{0}}{|\vec{p}|} Q_{0}\left(\frac{p_{0}}{|\vec{p}|}\right)\right] \delta_{n, 0}, \\
& G_{s}(p)=m_{D}^{2} \frac{p_{0}}{|\vec{p}|}\left[\left\{1-\left(\frac{p_{0}}{|\vec{p}|}\right)^{2}\right\} Q_{0}\left(\frac{p_{0}}{|\vec{p}|}\right)+\frac{p_{0}}{|\vec{p}|}\right] \delta_{n, 0}, \\
& Q_{0}(x)=\frac{1}{2} \ln \left|\frac{1+x}{1-x}\right|-i \frac{\pi}{2} \theta\left(1-x^{2}\right), \quad m_{D}^{2}=\frac{1}{4 \pi^{2}} N_{f} g^{2} \mu^{2},
\end{aligned}
$$

where $\theta$ is the Heaviside function and $\delta_{n, 0}$ signifies that the screening is effective only for the gluons with $n=0$. 
We can now write down the Dyson-Schwinger equation. We start with the equation for the $\Delta$-condensate. At high density (weak coupling), we can approximate the quark-gluon vertex with the bare ones and we have

$$
G(k)^{-1}-G_{0}(k)^{-1}=-i g^{2} X(D) \sum_{n} \int \frac{d^{4} p}{(2 \pi)^{4}} \Gamma^{\mu} G(p) \Gamma^{\nu} D_{\mu \nu}(q, n) .
$$

We have included the color factor $X(D)$ as in Equations (9) and the representation $D$ is either symmetric or antisymmetric depending on the channel that $G$ is in 11 Also we have defined $G_{0}$ as $G$ without the condensates, $q:=k-p$, and $\Gamma^{\mu}$ are

$$
\Gamma^{\mu}:=\left(\begin{array}{cccc}
0 & \sigma^{\mu} & 0 & 0 \\
\bar{\sigma}^{\mu} & 0 & 0 & 0 \\
0 & 0 & 0 & -\sigma^{\mu} \\
0 & 0 & -\bar{\sigma}^{\mu} & 0
\end{array}\right) .
$$

We can use (32), (35) and (40) to obtain the gap equation

$$
\begin{aligned}
\Delta_{ \pm}(k)= & i g^{2} X(D) \sum_{n} \int \frac{d^{4} p}{(2 \pi)^{4}} \\
& \operatorname{tr}\left[\sigma^{\mu}\left(\frac{\Delta_{+}(p) P_{R-}(p)}{p_{0}^{2}-(|\vec{p}|-\mu)^{2}-\Delta_{+}^{2}}+\frac{\Delta_{-}(p) P_{R+}(p)}{p_{0}^{2}-(|\vec{p}|+\mu)^{2}-\Delta_{-}^{2}}\right) \bar{\sigma}^{\nu} P_{L \pm}(k)\right] D_{\mu \nu}(q, n),
\end{aligned}
$$

where the trace is over the spinor indices and is taken to project out $\Delta_{ \pm}$from the $\Delta_{L}$-matrix in Equation (33). After some algebra, one obtains

$$
\begin{aligned}
\Delta_{+}\left(k_{0}\right) \approx & -i g^{2} X(D) \sum_{n} \int \frac{d^{4} p}{(2 \pi)^{4}} \\
& {\left[\frac{\Delta_{+}\left(p_{0}\right)}{p_{0}^{2}-(|\vec{p}|-\mu)^{2}-\Delta_{+}\left(p_{0}\right)^{2}}\left(\frac{1-(\hat{p} \cdot \hat{q})(\hat{k} \cdot \hat{q})}{q^{2}+(2 \pi n / L)^{2}+G_{s}(q)}+\frac{\frac{1}{2}+\frac{1}{2} \hat{p} \cdot \hat{k}}{q^{2}+(2 \pi n / L)^{2}+F_{s}(q)}\right)\right.} \\
+ & \left.+\frac{\Delta_{-}\left(p_{0}\right)}{p_{0}^{2}-(|\vec{p}|+\mu)^{2}-\Delta_{-}\left(p_{0}\right)^{2}}\left(\frac{1+(\hat{p} \cdot \hat{q})(\hat{k} \cdot \hat{q})}{q^{2}+(2 \pi n / L)^{2}+G_{s}(q)}+\frac{\frac{1}{2}-\frac{1}{2} \hat{p} \cdot \hat{k}}{q^{2}+(2 \pi n / L)^{2}+F_{s}(q)}\right)\right],
\end{aligned}
$$

where we have assumed that the gaps are functions only of $k_{0}$ or $p_{0}$. In deriving this equation, we have adopted the approximation, $q_{0} \ll|\vec{q}| \approx \mu$, so that $P_{\mu \nu}^{L} \approx \eta_{\mu \nu} \delta_{\mu, 0} \delta_{\nu, 0}$. The equation for $\Delta_{-}\left(k_{0}\right)$ is the same except that the two terms in the round brackets are exchanged. Note that only the first term in the equation of $\Delta_{+}\left(k_{0}\right)$ has the near-Fermisurface (infrared) divergence that is being cured by the formation of the condensate. Thus to the first approximation in large $\mu$, we can neglect the second term. Similar observation in the equation of $\Delta_{-}\left(k_{0}\right)$ results in the conclusion that this gap does not form at near the Fermi surface.

\footnotetext{
${ }^{11}$ The easiest way to see how this color factor comes in is to note that

$$
\sum_{a} T_{a b}^{\alpha} T_{c d}^{\alpha}=\frac{1}{2} X(\mathrm{symm})\left(\delta_{a b} \delta_{c d}+\delta_{a d} \delta_{b c}\right)+\frac{1}{2} X(\operatorname{asymm})\left(\delta_{a b} \delta_{c d}-\delta_{a d} \delta_{b c}\right) .
$$
}


Now as before, we set $\vec{p}=\vec{p}_{F}+\vec{l}_{\|}$, and approximate $\left|\vec{p}_{F}\right| \approx \mu, \hat{p}_{F} \cdot \hat{l}_{\|} \approx 1,|\vec{p}| \approx \mu+l_{\|}$ and $q^{2} \approx 2 \mu^{2}(1-\hat{p} \cdot \hat{k})$. Then the integration measure takes the form $\mu^{2} d p_{0} d l_{\|} d \cos \theta d \phi$ with $\cos \theta:=\hat{p} \cdot \hat{k}$. Since the integral is dominated by the region $\theta \approx 0$, we further approximate that $\hat{p} \cdot \hat{k} \approx 1$ and $\hat{p} \cdot \hat{q} \approx 0 \approx \hat{k} \cdot \hat{q}$ in the numerators of the integral, but not in the denominators. We Wick rotate $p_{0} \rightarrow i p_{0}$ and integrate over $l_{\|}$and $\phi$. The gap equation now takes the form

$$
\begin{aligned}
\Delta_{+}\left(k_{0}\right) & \approx-\frac{g^{2}}{8 \pi^{2}} X(D) \sum_{n} \int d p_{0} d \cos \theta\left(\frac{1}{1-\cos \theta+(1 / 2)\{2 \pi n /(\mu L)\}^{2}+G_{s}(q) /\left(2 \mu^{2}\right)}\right. \\
& \left.+\frac{1}{1-\cos \theta+(1 / 2)\{2 \pi n /(\mu L)\}^{2}+F_{s}(q) /\left(2 \mu^{2}\right)}\right) \frac{\Delta_{+}\left(p_{0}\right)}{\sqrt{p_{0}^{2}+\Delta_{+}\left(p_{0}\right)^{2}}}
\end{aligned}
$$

with the approximate form of the screenings

$$
F_{s}(q) \approx 2 m_{D}^{2} \delta_{n, 0}, \quad G_{s}(q) \approx \frac{\pi}{2} m_{D}^{2} \frac{q_{0}}{|\vec{q}|} \delta_{n, 0}
$$

From this equation, it is clear that for the symmetric channel $D=$ symm, we only have the trivial solution $\Delta_{+}=012$ We thus consider the antisymmetric channel from now on.

In Equation (45), the sum over $n$ and integral over $\theta$ can be carried out in a straightforward manner and yields

$$
\Delta_{+}\left(k_{0}\right) \approx \frac{g^{2}}{12 \pi^{2}} \frac{N_{c}+1}{2 N_{c}} \int d p_{0} \ln \left(\frac{\Lambda}{\left|k_{0}-p_{0}\right|}\right) \frac{\Delta_{+}\left(p_{0}\right)}{\sqrt{p_{0}^{2}+\Delta_{+}\left(p_{0}\right)^{2}}},
$$

where we have defined

$$
\Lambda:=2^{10} \sqrt{2} \pi^{4} N_{f}^{-5 / 2} g^{-5} \mu\{\sinh (\mu L) /(\mu L)\}^{4},
$$

and the part, $\{\cdots\}^{4}$, is the contribution from the sum over $n \neq 0$. The factor $\left|k_{0}-p_{0}\right|$ appearing in the logarithm comes from the Landau damping of $G_{s}(q)$ as in (46) and this effect was first discussed by Son [28]. We can follow Appendix B of Reference [28] to solve this equation and obtain

$$
\Delta_{+}\left(k_{0}\right)=\Delta_{0} \sin \left(\sqrt{\frac{12 \pi^{2}}{g^{2}} \frac{2 N_{c}}{N_{c}+1}} \ln \frac{\Lambda}{k_{0}}\right), \quad \text { with } \quad \Delta_{0}=\Lambda \exp \left[-\frac{\pi}{2} \sqrt{\frac{12 \pi^{2}}{g^{2}} \frac{2 N_{c}}{N_{c}+1}}\right] .
$$

Let us comment on this result. We first note that the gap vanishes in the 't Hooft limit. This is consistent with what we have concluded in the renormalization group analysis. Now, when $\mu L \ll 1$, we have $\sinh (\mu L) / \mu L \approx 1$, so the extra dimensional effect in $\Lambda$ disappears and the resulting expression for the gap coincide with the QCD result for $N_{c}=3$. (See, for example, Reference [1].) In the opposite limit where $\mu L \rightarrow \infty$, we have $\sinh (\mu L) \rightarrow \exp (\mu L) / 2$, so the extra dimensional effect contributes heavily and the gap grows with the parameter $\mu L$. As one can observe in Equation (45), this is because the infrared effect of the terms with $n \neq 0$ comes to be comparable to that of the $n=0$ term.

\footnotetext{
${ }^{12}$ If the flavor structure is included, this conclusion gets slightly more complicated. However, the fact that the antisymmetric channel dominates over the symmetric one does not change. See, for instance, Reference 33 .
} 
From Equation (49), we see that at $\mu L \sim 1 / g$, the gap is much larger than the size of the Fermi sphere itself and such a solution cannot be accepted, for the dynamics is no longer taking place near the Fermi surface. We therefore conclude that when $\mu L \sim 1 / g$, the gap does not form and the ground state simply is described by the Fermi liquid.

We now turn to the $\Sigma$-condensate, $\chi \mathrm{DW}$. The computation is almost identical to the previous case and we arrive at the equation similar to Equation (45) with the replacements $\Delta_{+} \rightarrow \Sigma_{+}$and $D \rightarrow \bullet$. There are, however, a few differences. The most important one is the range of the integration parameter $\theta$. This is restricted to the near infrared region, i.e., $\theta \approx 0$, because this is a forward scattering and the exchanged gluon should not be harder than the size of the gap or the momentum carried by the propagator $G(k)$. When the angle is set to small value, the $l_{\|}$component is about $\mu(1-\cos \theta)$, and the propagator carries the momentum approximately $\sqrt{p_{0}^{2}+\Sigma_{+}^{2}}$. We thus require

$$
\mu(1-\cos \theta) \leq \sqrt{p_{0}^{2}+\Sigma_{+}^{2}},
$$

and this inequality sets the upper limit on $\theta$. Now because of this kinematic restriction, when $N_{c} \approx 3$, the $\Delta$-condensate dominates over the $\Sigma$-condensate. Therefore, in the following, we consider the 't Hooft limit (with small $\lambda$ ). In this limit, the screening $F_{s}$ and $G_{s}$ is $1 / N_{c}$-suppressed, so we drop the screening terms from the gap equation. In the absence of the screening, the approximation, $q^{2} \approx 2 \mu^{2}(1-\cos \theta)$, has infrared problem when $n=0$. This means that $q_{0}^{2}$ cannot be neglected in this case and we must use $q^{2} \approx 2 \mu^{2}(1-\cos \theta)+$ $\left|k_{0}-p_{0}\right|^{2} \delta_{n, 0}$. We thus have the gap equation for the $\Sigma$-condensate

$$
\begin{aligned}
\Sigma_{+}\left(k_{0}\right)= & \frac{g^{2}}{8 \pi^{2}} \frac{N_{c}^{2}-1}{2 N_{c}} \sum_{n} \int d p_{0} d \cos \theta \\
& \frac{2}{1-\cos \theta+\left|k_{0}-p_{0}\right|^{2} /\left(2 \mu^{2}\right) \delta_{n, 0}+(1 / 2)\{2 \pi n /(\mu L)\}^{2}} \frac{\Sigma_{+}\left(p_{0}\right)}{\sqrt{p_{0}^{2}+\Sigma_{+}\left(p_{0}\right)^{2}}},
\end{aligned}
$$

where the integration range of $\theta$ is restricted as mentioned above.

Let us first consider the case with $n=0$. In this case, we can carry out the $\cos \theta$ integral with the restriction (50) and obtain

$$
\Sigma_{+}\left(k_{0}\right) \approx \frac{g^{2}}{8 \pi^{2}} \frac{N_{c}^{2}-1}{N_{c}} \int d p_{0} \ln \left(\frac{2 \mu \sqrt{p_{0}^{2}+\Sigma_{+}\left(p_{0}\right)^{2}}}{\left|k_{0}-p_{0}\right|^{2}}\right) \frac{\Sigma_{+}\left(p_{0}\right)}{\sqrt{p_{0}^{2}+\Sigma_{+}\left(p_{0}\right)^{2}}} .
$$

We can again solve this equation following Son [28] (also see [19]). In this case, the calculation is slightly different from Reference [28], so it is shown in Appendix B. The result is

$$
\Sigma_{+}\left(k_{0}\right)=\Sigma_{0} \cos \left(\sqrt{\frac{g^{2}}{4 \pi^{2}} \frac{N_{c}^{2}-1}{N_{c}}} \ln \frac{2 \mu}{k_{0}}\right), \quad \text { with } \quad \Sigma_{0}=2 \mu \exp \left[-\pi \sqrt{\frac{4 \pi^{2}}{g^{2}} \frac{N_{c}}{N_{c}^{2}-1}}\right] .
$$

This result agrees with Reference [13].

When we include the terms with $n \neq 0$, the logarithm term in Equation (52) gets augmented as

$$
\ln \frac{2 \mu \sqrt{p_{0}^{2}+\Sigma_{+}\left(p_{0}\right)^{2}}}{\left|k_{0}-p_{0}\right|^{2}}+4 \ln \frac{\sinh \left(\mu L \epsilon^{1 / 2}\right)}{\mu L \epsilon^{1 / 2}}
$$


where $\epsilon:=\sqrt{p_{0}^{2}+\Sigma_{+}^{2}} / \mu$. If $\mu L$ is not too large compared to $\epsilon^{1 / 2}$, then the second term in the above expression is small and the result (53) does not change. However, when $\mu L$ is so large that the second term yields dominant contribution $\sim \mu L \epsilon^{1 / 2}$, the integrand of the gap equation (52) takes the from proportional to $1 /\left(p_{0}^{2}+\Sigma_{+}^{2}\right)^{1 / 4}$, which is free of the infrared divergence even without the condensate $\Sigma$. This implies that the gap does not exist. To roughly estimate the value of $\mu L$ at which the crossover occurs, we approximate the first term in Equation (54) as $\ln \left(2 \mu / \Sigma_{0}\right)$ and the second term as $\mu L\left(\Sigma_{0} / \mu\right)^{1 / 2}$. Then we see that the second term becomes important when

$$
\mu L \gtrsim\left(\mu / \Sigma_{0}\right)^{1 / 2} \ln \left(\mu / \Sigma_{0}\right) \approx e^{1 / \sqrt{\lambda}} / \sqrt{\lambda}
$$

\section{Comments on the Strong Coupling Regime}

We have addressed the possibilities of the color superconductivity and the chiral density waves in the Sakai-Sugimoto model at finite density and explicitly carried out the computations in the weak coupling limit. As was stated in the introduction, the ultimate goal of this investigation is to obtain the quantitative behavior of the model in the weak and strong coupling regions, make qualitative comparison of the phase diagrams and gain insight into the QCD phase diagram which is still unsettled. We therefore comment on the strong coupling gravity background analysis of the model at finite density.

As suggested by Sakai and Sugimoto [15], the strong coupling analysis is done by taking the gravity background limit of the D4-branes while treating the D8-branes as probes, then the DBI-action of the probes are studied to obtain the spectrum of the low energy excitations at strong coupling. The generalization to the finite density has been discussed in References [34, 35, 36]. The phase diagram of the model in the space of temperature and chemical potential has been obtained by Horigome and Tanii [35. Let us briefly review their results. In the previous section, we have placed the D8- and $\overline{\mathrm{D} 8}$-branes at the antipodal points of the compactified circle. However, this is not necessary and in this gravity background analysis, the compactification radius is set to $R$ and the distance between the branes to $L$ with the range $0<L \leq \pi R$.

At finite temperature, in addition to the $x_{4}$-direction, the Euclidean time direction, $\tau$, is also compactified and the period of the time circle is identified with the inverse temperature. Horigome and Tanii consider the three known phases, first discovered by Aharony et al. [37] at zero density, and the spacetime configurations for the phases are illustrated in Figure 10. In the figure, the vertical $U$-axis represents the radial direction in 5,6,7,8 and 9 directions. The change in the background geometry of the phases from (a) to (b,c) is interpreted as the confinement/deconfinement phase transition by Aharony et al. The thinner lines and curves in the diagrams represent the $\mathrm{D} 8$ or $\overline{\mathrm{D} 8}$ branes and the change in the configuration from Diagram (b) to (c) is interpreted as the chiral symmetry restoration.

The on-shell DBI actions of the D8-branes with nonzero chemical potential have been obtained by Horigome and Tanii for each phase. For the configurations with the smooth U-shaped D8-branes, that is, for the diagrams (a) and (b) of Figure 10, they have found that the chemical potential must be constant along the radial $U$-direction and the actions for those configurations are independent of the chemical potential. Only for the parallel D8brane configuration of Diagram (c) has the non-trivial dependence on the chemical potential in its action. By comparing the on-shell actions at the various values of the temperature and 


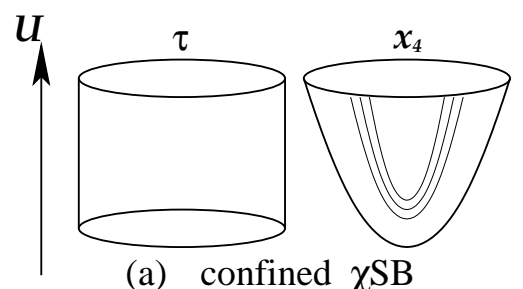

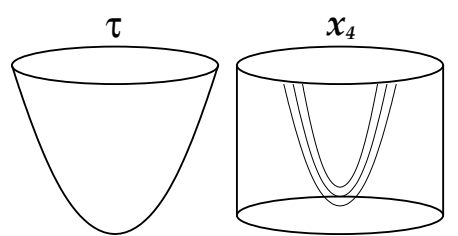

(b) deconfined $\chi \mathrm{SB}$

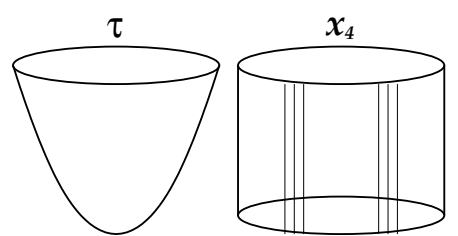

(c) deconfined $\chi \mathrm{S}$

Figure 10: The spacetime configurations of the three phases. The vertical axis $U$ is the radial direction in 5,6,7,8 and 9 directions. The thinner lines represent the stacks of D8 and $\overline{\mathrm{D} 8}$ branes. Diagram (a) is the low temperature confined phase. The chiral symmetry is broken in this phase. Diagram (b) is the high temperature deconfined phase and with $\chi \mathrm{SB}$. Diagram (c) is also the high temperature deconfined phase but with chiral symmetry restored.

the chemical potential, they determined the phase diagram which is schematically shown in Figure 1113

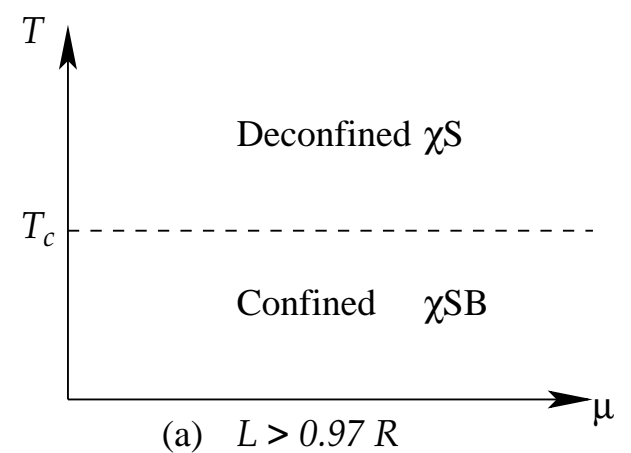

(a) $L>0.97 R$

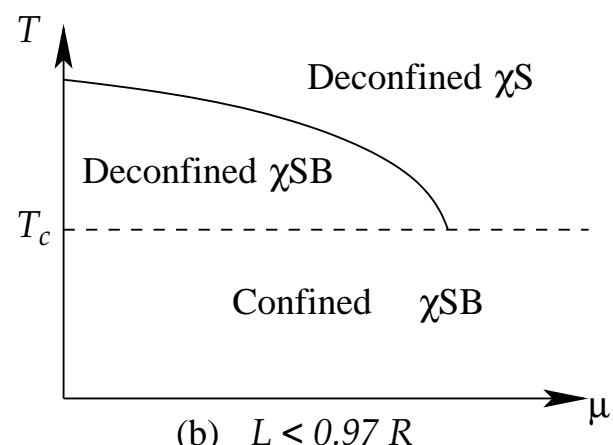

(b) $L<0.97 R$

Figure 11: The schematic phase diagram obtained by Horigome and Tanii. The temperature $T_{c}$ denotes the confinement/deconfinement phase transition temperature. The inter-D8 $\overline{\mathrm{D} 8}$ distance $L \simeq 0.97 R$ is the critical value where the deconfined phase with $\chi \mathrm{SB}$ exists.

The confinement/deconfinement phase transition line at $T=T_{c}$ is determined by the D4-background geometry and the D4 difference action in those phases scales as $N_{c}^{2}$ [37. This is expected to completely dominate the D8 probe actions, which scale as $N_{c} N_{f}$, so the confinement/deconfinement phase transition line is not affected by the value of the chemical potential 14 Therefore, Horigome and Tanii assume that the phase below the confinement/deconfinement line is in the configuration (a) of Figure 10, and compare the D8 actions of the configurations (b) and (c) in the deconfined D4-background geometry to obtain the phase structure above the confinement/deconfinement phase transition line. This is why the $\chi \mathrm{S} / \chi \mathrm{SB}$ phase transition line of the right panel in Figure 11 is terminated at $T=T_{c}$. When the inter-D8 $\overline{\mathrm{D} 8}$ distance $L$ is larger than $0.97 R$, it has been shown by Aharony et al. [37] that the phase represented in Diagram (b) of Figure 10 does not exist. Therefore, the phase diagram becomes rather structureless, as shown in the left panel of Figure 11.

\footnotetext{
${ }^{13}$ In Reference [35, the temperature and chemical potential are measured in different units. If measured in the same units, the chemical potential is asymptotically larger than the temperature by the factor of the 't Hooft coupling $\lambda$.

${ }^{14}$ In the original version of this e-print, we overlooked the dominance of the D4 action and compared only the D8 actions in the confined and deconfined phases, which was incorrect.
} 
The analysis of Horigome and Tanii assumes only three possible phases and this is similar to the simplified picture of QCD where one assumes only hadronic and quark-gluon plasma phases. In the latter, it is expected that when the chemical potential is raised several times $\Lambda_{Q C D}$ (at low temperature), the hadrons start to overlap and the quarks are shared by many hadrons. This implies the change in the degrees of freedom and since only the two phases are assumed, there should be a phase transition. Stated differently, in this simplified picture of QCD, we expect that a phase transition to occur even at zero temperature. See Figure 12 Though the physical setups are similar, we see that the phase diagrams in

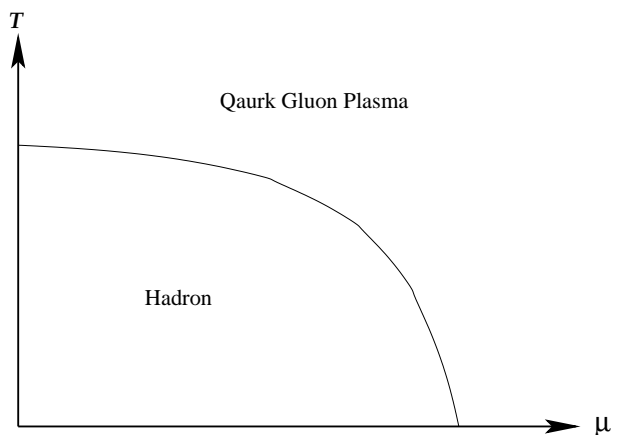

Figure 12: Expected phase diagram of the simplified QCD where only the hadronic and quark-gluon plasma phases are assumed to exist.

Figures 11 and 12 are qualitatively different. We suspect that the discrepancy stems from the large $N_{c}$ limit and the probe approximation in the Sakai-Sugimoto model. As we have explained, the confinement/deconfinement phase transition is determined by the D4 actions because the difference action scales with $N_{c}^{2}$ and dominates over the D 8 actions (with the chemical potential). It is unlikely that this picture can change in the probe approximation. Therefore, in order to see modifications in the confinement/deconfinement phase transition line, one should take into account of the D8-brane back reaction to the geometry.

It is also clear that we do not observe the color superconductivity or chiral density waves in the strong coupling analysis of Horigome and Tanii because these possibilities are simply not considered. To explore those exotic phases, one must come up with the corresponding stringy pictures of the branes and strings, and see if they are energetically preferred at any point in the $\mu-T$ parameter space. Such stringy pictures of quark matter are, so far, not clear to us 16 Nevertheless, we make some general remarks in this direction. First, we must remember that the relevant degrees of freedom at high density are particles and holes while the anti-particles are buried deep in the Dirac sea. Thus, if the U-shape configuration of the D8-branes describes the mesons, which are the pairs of particle and anti-particle, then we expect the configuration of the superconductor or chiral density waves to be different from the U-shape configuration because they are the pairs of particles and holes. Secondly, the Fermi sphere plays the essential role in high density QCD. Thus, the Fermi sphere must

\footnotetext{
${ }^{15}$ It is interesting to observe that the weakly coupled large $N_{c} \mathcal{N}=4$ super-Yang-Mills theory on three sphere and Type IIB supergravity on $\mathrm{AdS}_{5} \times S^{5}$ both have qualitatively the same phase structure as Figure 12 38, 39, 40. In these cases, however, the chemical potential is conjugate to the $U(1)$ subgroup of $S U(4)$ $R$-symmetry.

${ }^{16}$ The stringy configurations of baryons have been suggested in Refs. [41, 42] in which the nuclear matter phase has been discovered in the phase diagram.
} 
be encoded in the holographic picture somehow. Also, the holography is in the 't Hooft limit under which we do not expect non-gauge invariant quantities to survive. Therefore, it is likely that we do not observe the color superconducting phase and the relevant phase probably is the chiral density waves in the holographic theories.

Although Shuster and Son [14] settled that the color superconductivity dominates over the chiral density waves in the high density weakly coupled QCD (with $N_{c}=3$ ), it still is not known if this observation persists at the medium density strong coupling region. The Sakai-Sugimoto model in the usual 't Hooft limit is not likely to be able to address this problem but it is interesting to examine this in the limit with $N_{f} / N_{c}$ fixed. In this case, we can expect the effect of the color-flavor locking to be significant and also the competition between the D4 and D8 actions becomes non-trivial.

Finally, as we have mentioned in the introduction, the rich structure of the QCD phase diagram is partly due to the quark masses. As the authors of Reference [21] have already noted, the inclusion of the quark masses involves the tachyon that comes from the string stretching between the D8 and $\overline{\mathrm{D} 8}$ branes. This subject is being actively studied (for example, see Refs. [23, 24, 25, 29, 30]) and the resulting phase diagram is yet to be seen.

If the finite density holographic model turns out to capture the aspects of QCD, it would be very interesting because we can explore the region of the QCD phase diagram where the perturbative nor the numerical analysis is available.

\section{Acknowledgments}

I would like to thank the members of Racah Institute of Physics who provided valuable questions and comments. I benefited from Oren Bergman and Jacob Sonnenschein with regard to the strongly coupled aspects of the theory. I also would like to thank Andrei Kryjevski for educating me high density QCD in year 2004. This work was supported by the Golda Meir Post-Doctoral fellowship.

\section{A Some Formulas}

We adopt the convention of Wess and Bagger [26], except the definition of the Dirac spinor as in Equation (5). Many useful formulas can be found in Appendix B of the reference.

The propagators of the left- and right-handed quarks are respectively given as

$$
\frac{-i\left(p_{\nu}-\mu \delta_{\nu, 0}\right) \sigma_{\alpha \dot{\alpha}}^{\nu}}{\left(p_{\lambda}-\mu \delta_{\lambda, 0}\right)^{2}}, \text { and } \quad \frac{-i\left(p_{\nu}-\mu \delta_{\nu, 0}\right) \bar{\sigma}^{\nu \dot{\alpha} \alpha}}{\left(p_{\lambda}-\mu \delta_{\lambda, 0}\right)^{2}} \text {. }
$$

We define the charge conjugation matrix

$$
C:=\left(\begin{array}{cc}
i \sigma^{2} \bar{\sigma}^{0} & 0 \\
0 & i \bar{\sigma}^{2} \sigma^{0}
\end{array}\right),
$$

which satisfies $C^{-1} \gamma^{\nu} C=-\gamma^{\nu T}$ and $C^{-1}=C^{T}=-C$. 
The quark and anti-quark on-shell projectors are defined as

$$
\begin{aligned}
P_{L \pm}(p):=\frac{1}{2}\left(1 \pm \sigma^{0} \bar{\sigma}^{j} \hat{p}_{j}\right), & P_{R \pm}(p):=\frac{1}{2}\left(1 \pm \bar{\sigma}^{0} \sigma^{j} \hat{p}_{j}\right), \\
\bar{P}_{L \pm}(p):=\frac{1}{2}\left(1 \pm \hat{p}_{j} \bar{\sigma}^{j} \sigma^{o}\right), & \bar{P}_{R \pm}(p):=\frac{1}{2}\left(1 \pm \hat{p}_{j} \sigma^{j} \bar{\sigma}^{0}\right),
\end{aligned}
$$

where $\hat{p}:=\vec{p} /|\vec{p}|$. Notice that $P_{L \pm}=\bar{P}_{R \mp}$ and $P_{R \pm}=\bar{P}_{L \mp}$.

We list the useful formulas for inverting the inverse of the Nambu-Gor'kov propagator. For $n \times n$ matrices $A, B, C$ and $D$, we have

$$
\left(\begin{array}{ll}
A & B \\
C & D
\end{array}\right)^{-1}=\left(\begin{array}{cc}
A^{-1}+A^{-1} B S_{A}^{-1} C A^{-1} & -A^{-1} B S_{A}^{-1} \\
-S_{A}^{-1} C A^{-1} & S_{A}^{-1}
\end{array}\right),
$$

provided that the matrices $A$ and $S_{A}:=D-C A^{-1} B$ are invertible. We also have

$$
\left(\begin{array}{ll}
A & B \\
C & D
\end{array}\right)^{-1}=\left(\begin{array}{cc}
-C^{-1} D S_{C}^{-1} & C^{-1}+C^{-1} D S_{C}^{-1} A C^{-1} \\
S_{C}^{-1} & -S_{C}^{-1} A C^{-1}
\end{array}\right),
$$

provided that $C$ and $S_{C}:=B-A C^{-1} D$ are invertible. Other convenient formulas are the following.

$$
\begin{gathered}
\left(p_{\nu}-\mu \delta_{\nu, 0}\right) \sigma^{\nu}=\left(p_{0}+|\vec{p}|-\mu\right) \sigma^{0} P_{R+}+\left(p_{0}-|\vec{p}|-\mu\right) \sigma^{0} P_{R-}, \\
\left(p_{\nu}-\mu \delta_{\nu, 0}\right) \bar{\sigma}^{\nu}=\left(p_{0}+|\vec{p}|-\mu\right) \bar{\sigma}^{0} P_{L+}+\left(p_{0}-|\vec{p}|-\mu\right) \bar{\sigma}^{0} P_{L-} . \\
\left\{\left(p_{\nu}-\mu \delta_{\nu, 0}\right) \sigma^{\nu}\right\}^{-1}=\frac{\bar{\sigma}^{0} P_{L+}}{p_{0}-|\vec{p}|-\mu}+\frac{\bar{\sigma}^{0} P_{L-}}{p_{0}+|\vec{p}|-\mu}, \\
\left\{\left(p_{\nu}-\mu \delta_{\nu, 0}\right) \bar{\sigma}^{\nu}\right\}^{-1}=\frac{\sigma^{0} P_{R+}}{p_{0}-|\vec{p}|-\mu}+\frac{\sigma^{0} P_{R-}}{p_{0}+|\vec{p}|-\mu} . \\
P_{L \pm} \sigma^{0} P_{R \pm}=0, \quad P_{L \pm} \sigma^{0} P_{R \mp}=\sigma^{0} P_{R \mp} \\
P_{R \pm} \bar{\sigma}^{0} P_{L \pm}=0, \quad P_{R \pm} \bar{\sigma}^{0} P_{L \mp}=\bar{\sigma}^{0} P_{L \mp} .
\end{gathered}
$$

\section{B Solving Gap Equation}

We solve Equation (52), following References [28, 19]. We split the integration range into, $0<p_{0}<k_{0}$ and $k_{0}<p_{0}<2 \mu$. Then the dominant contributions in the logarithms of the integrand are $\ln \frac{2 \mu p_{0}}{k_{0}^{2}}=2 \ln \frac{2 \mu}{k_{0}}-\ln \frac{2 \mu}{p_{0}}$ for the former integration region and $\ln \frac{2 \mu}{p_{0}}$ for the latter. We introduce the parameters

$$
x:=\ln \frac{2 \mu}{k_{0}}, \quad y:=\ln \frac{2 \mu}{p_{0}}, \quad x_{0}:=\ln \frac{2 \mu}{\Sigma_{0}},
$$

where $\Sigma_{0}:=\Sigma(0)$. Then the gap equation is cast into the form

$$
\Sigma(x)=\frac{g^{2}}{8 \pi^{2}} \frac{N_{c}^{2}-1}{N_{c}}\left(2 x \int_{x}^{x_{0}} \Sigma(y) d y-\int_{x}^{x_{0}} y \Sigma(y) d y+\int_{0}^{x} y \Sigma(y) d y\right) .
$$


Note that we have $\Sigma\left(x_{0}\right)=-\Sigma(0)$. We can take the derivative with respect to $x$ twice to get

$$
\Sigma^{\prime \prime}=-\frac{g^{2}}{4 \pi^{2}} \frac{N_{c}^{2}-1}{N_{c}} \Sigma
$$

and the appropriate solution is

$$
\Sigma(x)=\Sigma_{0} \cos \left(x \sqrt{\frac{g^{2}}{4 \pi^{2}} \frac{N_{c}^{2}-1}{N_{c}}}\right), \quad \text { with } \quad x_{0}=\pi \sqrt{\frac{4 \pi^{2}}{g^{2}} \frac{N_{c}}{N_{c}^{2}-1}} .
$$

From the definition of $x_{0}$, we obtain

$$
\Sigma_{0}=2 \mu \exp \left[-\pi \sqrt{\frac{4 \pi^{2}}{g^{2}} \frac{N_{c}}{N_{c}^{2}-1}}\right] .
$$




\section{References}

[1] K. Rajagopal and F. Wilczek, The condensed matter physics of QCD, arXiv:hep-ph/0011333.

[2] M. A. Stephanov, QCD phase diagram: An overview, PoS LAT2006, 024 (2006) arXiv:hep-lat/0701002.

[3] Y. Nambu and G. Jona-Lasinio, Dynamical model of elementary particles based on an analogy with superconductivity. I, Phys. Rev. 122, 345 (1961).

[4] Y. Nambu and G. Jona-Lasinio, Dynamical model of elementary particles based on an analogy with superconductivity. II, Phys. Rev. 124, 246 (1961).

[5] T. Hatsuda and T. Kunihiro, QCD phenomenology based on a chiral effective Lagrangian, Phys. Rept. 247, 221 (1994) arXiv:hep-ph/9401310.

[6] I. A. Shovkovy, Two lectures on color superconductivity, Found. Phys. 35, 1309 (2005) arXiv:nucl-th/0410091.

[7] M. Buballa, NJL model analysis of quark matter at large density, Phys. Rept. 407, 205 (2005) arXiv:hep-ph/0402234.

[8] M. G. Alford, Color superconductivity in ultra-dense quark matter, arXiv:hep-lat/0610046.

[9] D. Bailin and A. Love, Superfluidity And Superconductivity In Relativistic Fermion Systems, Phys. Rept. 107, 325 (1984).

[10] J. Bardeen, L. N. Cooper and J. R. Schrieffer, Theory Of Superconductivity, Phys. Rev. 108, 1175 (1957).

[11] M. G. Alford, K. Rajagopal and F. Wilczek, Color-flavor locking and chiral symmetry breaking in high density QCD, Nucl. Phys. B 537, 443 (1999) arXiv:hep-ph/9804403.

[12] R. Casalbuoni and R. Gatto, Effective theory for color-flavor locking in high density QCD, Phys. Lett. B 464, 111 (1999) [arXiv:hep-ph/9908227].

[13] D. V. Deryagin, D. Y. Grigoriev and V. A. Rubakov, Standing wave ground state in high density, zero temperature QCD at large N(c), Int. J. Mod. Phys. A 7 (1992) 659 .

[14] E. Shuster and D. T. Son, On finite-density QCD at large N(c), Nucl. Phys. B 573, 434 (2000) arXiv:hep-ph/9905448.

[15] T. Sakai and S. Sugimoto, Low energy hadron physics in holographic QCD, Prog. Theor. Phys. 113, 843 (2005) arXiv:hep-th/0412141.

[16] T. Sakai and S. Sugimoto, More on a holographic dual of QCD, Prog. Theor. Phys. 114, 1083 (2006) arXiv:hep-th/0507073.

[17] J. Polchinski, Effective Field Theory And The Fermi Surface, arXiv:hep-th/9210046. 
[18] D. K. Hong, Aspects of high density effective theory in $Q C D$, Nucl. Phys. B 582, 451 (2000) arXiv:hep-ph/9905523.

[19] B. Y. Park, M. Rho, A. Wirzba and I. Zahed, Dense QCD: Overhauser or BCS pairing?, Phys. Rev. D 62, 034015 (2000) [arXiv:hep-ph/9910347].

[20] E. Witten, Anti-de Sitter space, thermal phase transition, and confinement in gauge theories, Adv. Theor. Math. Phys. 2, 505 (1998) arXiv:hep-th/9803131.

[21] S. Sugimoto and K. Takahashi, QED and string theory, JHEP 0404, 051 (2004) arXiv:hep-th/0403247.

[22] E. Antonyan, J. A. Harvey, S. Jensen and D. Kutasov, NJL and QCD from string theory, arXiv:hep-th/0604017.

[23] O. Bergman, S. Seki and J. Sonnenschein, Quark mass and condensate in HQCD, JHEP 0712, 037 (2007) arXiv:0708.2839 [hep-th]].

[24] A. Dhar and P. Nag, Sakai-Sugimoto model, Tachyon Condensation and Chiral symmetry Breaking, JHEP 0801, 055 (2008) arXiv:0708.3233 [hep-th]].

[25] A. Dhar and P. Nag, Tachyon condensation and quark mass in modified SakaiSugimoto model, arXiv:0804.4807 [hep-th].

[26] J. Wess and J. Bagger, Supersymmetry and Supergravity, 2nd Ed., Princeton University Press, 1992

[27] N. J. Evans, S. D. H. Hsu and M. Schwetz, An effective field theory approach to color superconductivity at high quark density, Nucl. Phys. B 551, 275 (1999) arXiv:hep-ph/9808444].

[28] D. T. Son, Superconductivity by long-range color magnetic interaction in high-density quark matter, Phys. Rev. D 59, 094019 (1999) [arXiv:hep-ph/9812287].

[29] O. Aharony and D. Kutasov, Holographic Duals of Long Open Strings, arXiv:0803.3547 [hep-th].

[30] K. Hashimoto, T. Hirayama, F. L. Lin and H. U. Yee, Quark Mass Deformation of Holographic Massless QCD, arXiv:0803.4192 [hep-th].

[31] K. Rajagopal and E. Shuster, On the applicability of weak-coupling results in high density QCD, Phys. Rev. D 62, 085007 (2000) arXiv:hep-ph/0004074.

[32] M. Le Bellac, Thermal Field Theory, Cambridge University Press, 1996

[33] I. A. Shovkovy and L. C. R. Wijewardhana, On gap equations and color flavor locking in cold dense QCD with three massless flavors, Phys. Lett. B 470, 189 (1999) arXiv:hep-ph/9910225].

[34] K. Y. Kim, S. J. Sin and I. Zahed, Dense hadronic matter in holographic QCD, arXiv:hep-th/0608046. 
[35] N. Horigome and Y. Tanii, Holographic chiral phase transition with chemical potential, JHEP 0701, 072 (2007) arXiv:hep-th/0608198.

[36] A. Parnachev and D. A. Sahakyan, Photoemission with chemical potential from QCD gravity dual, Nucl. Phys. B 768, 177 (2007) arXiv:hep-th/0610247].

[37] O. Aharony, J. Sonnenschein and S. Yankielowicz, A holographic model of deconfinement and chiral symmetry restoration, Annals Phys. 322, 1420 (2007) [arXiv:hep-th/0604161].

[38] D. Yamada and L. G. Yaffe, Phase diagram of $N=4$ super-Yang-Mills theory with R-symmetry chemical potentials, JHEP 0609, 027 (2006) arXiv:hep-th/0602074.

[39] A. Chamblin, R. Emparan, C. V. Johnson and R. C. Myers, Charged AdS black holes and catastrophic holography, Phys. Rev. D 60, 064018 (1999) arXiv:hep-th/9902170.

[40] M. Cvetic and S. S. Gubser, Phases of R-charged black holes, spinning branes and strongly coupled gauge theories, JHEP 9904, 024 (1999) arXiv:hep-th/9902195].

[41] O. Bergman, G. Lifschytz and M. Lippert, Holographic Nuclear Physics, JHEP 0711, 056 (2007) arXiv:0708.0326 [hep-th]].

[42] M. Rozali, H. H. Shieh, M. Van Raamsdonk and J. Wu, Cold Nuclear Matter In Holographic QCD, JHEP 0801, 053 (2008) arXiv:0708.1322 [hep-th]]. 